\title{
Cryptanalysis of McEliece Cryptosystem Based on Algebraic Geometry Codes and their Subcodes
}

\author{
Alain Couvreur*1, Irene Márquez-Corbella*†2, and Ruud Pellikaan ${ }^{3}$ \\ ${ }^{1}$ INRIA, Laboratoire LIX, École Polytechnique \& CNRS UMR 7161, Université \\ Paris-Saclay. École Polytechnique, 91128 Palaiseau Cedex - France, \\ alain.couvreur@lix.polytechnique.fr \\ ${ }^{2}$ University of La Laguna, Dept. Maths., Stats. and O.R., 38271 La Laguna - Spain, \\ imarquec@ull.es \\ ${ }^{3}$ Eindhoven University of Technology P.O. Box 513, 5600 MB Eindhoven, \\ g.r.pellikaan@tue.nl
}

September 11, 2018

\begin{abstract}
We give polynomial time attacks on the McEliece public key cryptosystem based either on algebraic geometry (AG) codes or on small codimensional subcodes of AG codes. These attacks consist in the blind reconstruction either of an Error Correcting Pair (ECP), or an Error Correcting Array (ECA) from the single data of an arbitrary generator matrix of a code. An ECP provides a decoding algorithm that corrects up to $\frac{d^{*}-1-g}{2}$ errors, where $d^{*}$ denotes the designed distance and $g$ denotes the genus of the corresponding curve, while with an ECA the decoding algorithm corrects up to $\frac{d^{*}-1}{2}$ errors. Roughly speaking, for a public code of length $n$ over $\mathbb{F}_{q}$, these attacks run in $O\left(n^{4} \log (n)\right)$ operations in $\mathbb{F}_{q}$ for the reconstruction of an ECP and $O\left(n^{5}\right)$ operations for the reconstruction of an ECA. A probabilistic shortcut allows to reduce the complexities respectively to $O\left(n^{3+\varepsilon} \log (n)\right)$ and $O\left(n^{4+\varepsilon}\right)$. Compared to the previous known attack due to Faure and Minder, our attack is efficient on codes from curves of arbitrary genus. Furthermore, we investigate how far these methods apply to subcodes of AG codes.
\end{abstract}

\section{Introduction}

Most of the commonly used public key cryptosystems are based only on two problems: the hardness of factoring or the presumed intractability of the discrete logarithm problem. However, nothing ensures that the intractability of these problems will remain true for the foreseeable future. In particular, we should notice that all these classical number theory problems would be broken through P. Shor's quantum factorization algorithm [40] in the case a quantum computer would come to exist. Thus, the cryptographic community should look for alternative cryptosystems namely Post-quantum cryptography. Code-based Cryptography, together with lattice-based cryptography, multivariate cryptography and hash-based cryptography are the principal available techniques for Post-quantum cryptography (for instance see [2]).

\footnotetext{
*Partially funded by ANR grant ANR-15-CE39-0013-01 "Manta" and by the Commission of the European community the Horizon 2020 program ICT-645622 "PQCRYPTO".

${ }^{\dagger}$ Partially funded by Spanish grants MTM2016-80659-P and MTM2016-80659-P
} 
In the late seventies, McEliece [28] introduced the first code based public-key cryptosystem whose security reposes on the hardness of decoding a random linear code. Compared to publickey schemes based on integer factorization (like RSA) or discrete logarithm, McEliece not only is resistant, so far, to attacks by quantum computers, but also presents faster encryption and decryption schemes. However, due to the large size of the keys required to have a good security level, it is rarely used in practice. Nevertheless, note that recent proposals based on quasi-cyclic MDPC codes [30] allow compact keys of around 10000 bits for 128 bits of security, which makes such proposal competitive with RSA.

The original proposal [28], which remains unbroken, was based on binary Goppa codes. Later, several alternatives families with a higher correction capacity were proposed in order to reduce the key size. For instance, Generalized Reed-Solomon codes [32, subcodes of them [1] and Binary Reed-Muller codes [41, (note that this list is not exhaustive). All of these schemes are subject to polynomial or sub-exponential time attacks [29,42,46].

Another attempt, suggested by Janwa and Moreno [17] was to use algebraic geometry (AG) codes, their subfield subcodes or concatenated AG codes. Take notice that:

- The case of codes on curves of genus 0 was broken by Sidelnikov and Shestakov [42]. For curves of genus 1 and 2 it was broken by Faure and Minder [13], but this attack has several drawbacks which makes it impossible to generalize to higher genera. For instance, the curve is required to be hyperelliptic, which is non generic for $g>2$ and the attack involves the computation of minimum-weight codewords whose cost is exponential in the genus of the curve.

- In [39], Sendrier pointed out the inherent weakness of concatenated codes for public key cryptography. Thus any proposal using concatenation should be avoided.

- In [27], the authors proved that the structure of the curve can be recovered from the only knowledge of a generator matrix of the code. Unfortunately, the efficient construction of a decoding algorithm from the obtained code's representation is still lacking. Thus, this result does not lead to an efficient attack.

In this article, we use another approach to attack the McEliece scheme based on AG codes. Our attack is inspired by the attacks developed in [5, 6, 9, 10] called filtration attacks. Such attack uses the fact that the computation of some Schur products permits to distinguish AG codes from random ones. Moreover, this distinguisher can be used to compute an interesting filtration of the code used as a public key. Compared to the previous filtration attacks which allowed to recover completely the structure of the public key, the present attack is not actually a key recovery attack. In particular, we do not compute the structure of the curve and the divisors providing the public code and we show that such computations are not necessary. Indeed, it is possible to stay in the realm of $\mathbb{F}_{q}^{n}$ and its subspaces in order to compute all the necessary data to get an efficient decoding algorithm for the AG code used as a public key. More precisely, we show how to use filtration attack techniques in order to compute an error correcting pair (see [33, 36]) allowing to correct up to $\frac{d^{*}-1-g}{2}$ errors, where $d^{*}$ denotes the designed distance. These techniques can be pushed forward in order to compute an error correcting array [21] or equivalently a well behaving sequence [16] allowing to correct up to $\frac{d^{*}-1}{2}$ errors. The cost of this reconstruction is in $O\left(n^{4} \log (n)\right)$ operations on the base field for the reconstruction of an error correcting pair and $O\left(n^{5}\right)$ for the reconstruction of an error correcting array. A probabilistic shortcut permits one to reduce these complexities to $O\left(n^{3+\varepsilon}\right)$ and $O\left(n^{4+\varepsilon}\right)$ respectively. Finally, it is worth noting that many computations done in this filtration attack are very similar to those presented in a very different context by Khuri-Makdisi in [19] in order to perform effective computations on Jacobians of curves. 
The attack presented in this article is proved to be efficient for almost any algebraic geometry code. It should be emphasized that for some codes on curves of large genus $g$ and whose length satisfies $2 g<n<6 g$, the proofs of efficiency of the attack do not hold. Let us emphasize that this does not mean that the attack will fail for such codes but only that we have no mathematical proof for the efficiency of such cryptanalysis methods. On the other hand, we show that there are several ways to extend the attack and we doubt that it would be possible to provide a large family of codes for which our attack would be guaranteed to fail.

Outline of the article. Section 1 lists notation used in the article and introduces some necessary material for the attack. Next, we deal with two operations on codes: the Schur product introduced in Section 2 and a new operation which we have called the $s$-closure, defined in Section 3. The first one will be essential to attack the McEliece scheme based on AG codes while the second one will be crucial to attack McEliece scheme based on subcodes of AG codes. Section 4 is devoted to the notion of error-correcting pairs (ECP) and error-correcting arrays (ECA) which provide efficient decoding algorithms for AG codes. Section 5 provides a detailed exposition of all the results needed for our attack. In particular we show how to compute an error correcting pair or an error correcting array of a given code only by computing Schur products and performing Gaussian elimination. Finally, Section 6 indicates how all the previous techniques may be used to create an attack of the McEliece scheme based on AG codes and Section 7 deals with the case of subcodes of AG codes.

Note. A part of the material of this article was presented at the conferences ISIT1 2014 and ICMCTA2 2014 and published in [7, 8). The present article provides a long version including detailed proofs which were absent in the proceedings due to space reasons. It also includes new results since the proceedings articles only considered the reconstruction of error correcting pairs while we discuss here the reconstruction of error correcting arrays.

\section{Prerequisites on curves and algebraic geometry codes}

This section contains a brief summary of algebraic curves and algebraic geometry (AG) codes to set up notation and terminology. For a fuller treatment we refer the reader to [44,45].

\subsection{Curves}

Let $\mathcal{X}$ denote a smooth projective geometrically connected curve over a finite field $\mathbb{F}_{q}$ and let $g$ denote the genus of $\mathcal{X}$. The function field of the curve $\mathcal{X}$ with field of constants $\mathbb{F}_{q}$ is denoted by $\mathbb{F}_{q}(\mathcal{X})$ and its elements are called rational functions. Given a place $P$ of $\mathbb{F}_{q}(\mathcal{X})$, its degree is denoted as $\operatorname{deg} P$ and the valuation at $P$ of any $f \in \mathbb{F}_{q}(\mathcal{X})^{\times}$, is denoted by $\mathrm{v}_{P}(f)$. We use the convention $\mathrm{v}_{p}(0)=\infty$.

A divisor $E$ on $\mathcal{X}$ is a formal sum of places $E=\sum_{P \in \mathcal{X}} n_{P} P$ with $n_{P} \in \mathbb{Z}$. The degree of $E$ is the integer $\operatorname{deg}(E):=\sum_{P \in \mathcal{X}} n_{P} \operatorname{deg}(P)$ and the support of $E$, is the set of places with $n_{P} \neq 0$. If all coefficients $n_{P}$ are nonnegative, $E$ is an effective divisor, denoted by $E \geqslant 0$. Next, we denote by $E \geqslant F$ when $E-F \geqslant 0$. This defines a partial order on the group of divisors.

Let $f \in \mathbb{F}_{q}(\mathcal{X}) \backslash\{0\}$, the divisor of $f$ is denoted by $(f)$. Given a divisor $E$ on $\mathcal{X}$, the corresponding Riemann Roch space is denoted by $L(E)$ and is defined as follows

$$
L(E)=\left\{f \in \mathbb{F}_{q}(\mathcal{X}) \mid f=0 \text { or }(f)+E \geqslant 0\right\} .
$$

\footnotetext{
${ }^{1}$ IEEE International Symposium on Information Theory

${ }^{2}$ International Castle Meeting on Coding Theory and their Applications
} 


\subsection{Weierstrass gaps}

Gaps and non gaps are fundamental notions in this article. For this reason we remind several very usual properties of these objects. See [21] for further details.

Definition 1. Let $E$ be a divisor on $\mathcal{X}$ and $P$ be a rational point. A positive integer $i$ is called an $E$-gap at $P$ if $L(E+i P)=L(E+(i-1) P)$. Otherwise, $i$ is an $E$ non-gap at $P$. In case $E=0$, we just say gap and non-gap, respectively.

The following statement lists many elementary properties of gap and non-gap sets.

Proposition 1. (1) If $i \geqslant-\operatorname{deg}(E)+2 g$ then $i$ is an $E$ non-gap at $P$.

(2) The E-gaps at $P$ lie in the interval $[-\operatorname{deg}(E),-\operatorname{deg}(E)+2 g-1]$ and the number of gaps is exactly equal to $\mathrm{g}$.

(3) If $\alpha$ is an $F$ non-gap at $P$ and $\beta$ is an $E$ non-gap at $P$. Then, $\alpha+\beta$ is an $(F+E)$ non-gap at $P$. In particular if $\alpha$ is a non-gap at $P$ and $\beta$ is an $E$ non-gap at $P$. Then, $\alpha+\beta$ is an E non-gap at $P$.

(4) Let $\left(\alpha_{i}\right)_{i \in \mathbb{N}}$ be the non-gap sequence at $P$ and $\left(\beta_{j}\right)_{j \in \mathbb{N}}$ be the $E$ non-gap sequence at $P$. Then,

$$
j=\operatorname{dim} L\left(E+\beta_{j} P\right)=\operatorname{dim} L\left(E+\left(\beta_{j-1}\right) P\right)+1 .
$$

Thus, $j-1 \leqslant \operatorname{deg}(E)+\beta_{j} \leqslant j+g-1$. Moreover, $\operatorname{deg}(E)+\beta_{j}=j+g-1$ if $\operatorname{deg}(E)+\beta_{j}>2 g-1$, i.e. $j>$ g. Similarly $i-1 \leqslant \alpha_{i} \leqslant i+g-1$. Thus, $\alpha_{i}=i+g-1$ if $i>g$.

\subsection{Algebraic geometry codes}

We assume that the reader is aware of basic notions of coding theory and refer to [24] for further details. Below we remind some basic notions on algebraic geometry codes and refer the reader to [4, 45.

Given an $n$-tuple $\mathcal{P}=\left(P_{1}, \ldots, P_{n}\right)$ of pairwise distinct $\mathbb{F}_{q}$-rational points of $\mathcal{X}$, we denote by $D_{\mathcal{P}}$ the divisor $D_{\mathcal{P}}:=P_{1}+\cdots+P_{n}$. Let $E$ be a divisor of $\mathcal{X}$ with disjoint support from $P$, then the evaluation map

$$
\operatorname{ev}_{\mathcal{P}}:\left\{\begin{array}{ccc}
L(E) & \rightarrow & \mathbb{F}_{q}^{n} \\
f & \mapsto & \left(f\left(P_{1}\right), \ldots, f\left(P_{n}\right)\right)
\end{array}\right.
$$

is well-defined.

Definition 2. Let $\mathcal{P}=\left(P_{1}, \ldots, P_{n}\right)$ be an $n$-tuple of mutually distinct $\mathbb{F}_{q}$-rational points of the curve $\mathcal{X}$ and let $E$ be a divisor of $\mathcal{X}$ with disjoint support from $D_{\mathcal{P}}$. Then, the algebraic geometry (AG) code $\mathcal{C}_{L}(\mathcal{X}, \mathcal{P}, E)$ of length $n$ over $\mathbb{F}_{q}$ is the image of $L(E)$ under the evaluation map ev $v_{\mathcal{P}}$, that is

$$
\mathcal{C}_{L}(\mathcal{X}, \mathcal{P}, E)=\left\{\operatorname{ev}_{\mathcal{P}}(f) \mid f \in L(E)\right\} .
$$

From now on, the dimension of a linear code $C$ will be denoted by $k(C)$ and its minimum distance by $d(C)$. Let $\mathcal{X}, \mathcal{P}$ and $E$ be respectively a smooth projective geometrically connected curve over $\mathbb{F}_{q}$, an $n$-tuple of $\mathbb{F}_{q}$-rational points of $\mathcal{X}$ and a divisor on $\mathcal{X}$. Let us remind some well-known statements.

Theorem 2 ([44, Theorem 2.2.2]). If $\operatorname{deg}(E)<n$, then

$$
\begin{aligned}
& k\left(\mathcal{C}_{L}(\mathcal{X}, \mathcal{P}, E)\right) \geqslant \operatorname{deg}(E)+1-g \\
& d\left(\mathcal{C}_{L}(\mathcal{X}, \mathcal{P}, E)\right) \geqslant n-\operatorname{deg}(E) .
\end{aligned}
$$

Moreover, if $n>\operatorname{deg}(E)>2 g-2$, then $k\left(\mathcal{C}_{L}(\mathcal{X}, \mathcal{P}, E)\right)=\operatorname{deg}(E)-g+1$. 
Theorem 3 ([44, Proposition 2.2.10]). There exists a differential form $\omega$ with a simple pole and residue 1 at $P_{j}$ for all $j \in\{1, \ldots, n\}$. Let $K$ be the divisor of $\omega$, then

$$
\mathcal{C}_{L}(\mathcal{X}, \mathcal{P}, E)^{\perp}=\mathcal{C}_{L}\left(\mathcal{X}, \mathcal{P}, E^{\perp}\right),
$$

where $E^{\perp}:=D_{\mathcal{P}}-E+K$ and $\operatorname{deg}\left(E^{\perp}\right)=n-\operatorname{deg}(E)+2 g-2$.

\section{Schur product of codes}

The notion of Schur product of codes was first introduced in coding theory for decoding [33]. Next, this apparently trivial operation turned out to have many other applications such as cryptanalysis, multiparty computation, secret sharing or construction of lattices. Many of these applications are summarized in [38, §4].

Definition 3. The Schur product is the component wise product on $\mathbb{F}_{q}^{n}$ : given two elements a and $\mathbf{b}$ in $\mathbb{F}_{q}^{n}$,

$$
\mathbf{a} * \mathbf{b}:=\left(a_{1} b_{1}, \ldots, a_{n} b_{n}\right) .
$$

For two codes $A, B \subseteq \mathbb{F}_{q}^{n}$, their Schur product is the code $A * B$ defined as

$$
A * B:=\operatorname{Span}_{\mathbb{F}_{q}}\{\mathbf{a} * \mathbf{b} \mid \mathbf{a} \in A \text { and } \mathbf{b} \in B\} .
$$

For $B=A$, then $A * A$ is denoted as $A^{(2)}$ and, we define $A^{(t)}$ by induction for any positive integer $t$.

\subsection{Relation with the canonical inner product}

Take notice that the Schur product should not be confused with the standard inner product which is defined as

$$
\langle\mathbf{a}, \mathbf{b}\rangle:=\sum_{i=1}^{n} a_{i} b_{i} .
$$

However, the two notions are related by the following elementary adjunction principle:

$$
\forall \mathbf{a}, \mathbf{b}, \mathbf{c} \in \mathbb{F}_{q}^{n}, \quad\langle\mathbf{a} * \mathbf{b}, \mathbf{c}\rangle=\langle\mathbf{a}, \mathbf{b} * \mathbf{c}\rangle=\sum_{i=1}^{n} a_{i} b_{i} c_{i} .
$$

An interesting consequence of this relation between the Schur product and the canonical inner product is the following statement which is very useful in what follows.

Lemma 4. Let $A$ and $B$ be two codes in $\mathbb{F}_{q}^{n}$. Then

$$
\left\{\mathbf{z} \in \mathbb{F}_{q}^{n} \mid \mathbf{z} * A \subseteq B\right\}=\left(A * B^{\perp}\right)^{\perp} .
$$

Proof. It is easily seen that

$$
\begin{aligned}
\mathbf{z} * A \subseteq B & \Leftrightarrow\langle\mathbf{z} * \mathbf{a}, \mathbf{b}\rangle=0, \forall \mathbf{a} \in A \text { and } \forall \mathbf{b} \in B^{\perp} \\
& \Leftrightarrow\langle\mathbf{z}, \mathbf{a} * \mathbf{b}\rangle=0, \forall \mathbf{a} \in A \text { and } \forall \mathbf{b} \in B^{\perp} \Leftrightarrow \mathbf{z} \in\left(A * B^{\perp}\right)^{\perp} .
\end{aligned}
$$




\subsection{Schur product of algebraic geometry codes}

An interesting aspect of the Schur product is that the evaluation map ev $\mathcal{P}$ introduced in Section 1.3 arises from a morphism of algebras. More precisely, let $\mathcal{O}_{\mathcal{P}}$ be the subring of $\mathbb{F}_{q}(\mathcal{X})$ of functions regular at $P_{1}, \ldots, P_{n}$ then, the map ev $\mathcal{P}: \mathcal{O}_{\mathcal{P}} \rightarrow \mathbb{F}_{q}^{n}$ is a morphism of algebras since for all $f, g \in \mathcal{O}_{\mathcal{P}}$, we have $\operatorname{ev}(f g)=\operatorname{ev}(f) * \operatorname{ev}(g)$. Therefore, many arithmetic properties of the function field $\mathbb{F}_{q}(\mathcal{X})$ can be understood in terms of AG codes thanks to the Schur product.

To understand the behaviour of Schur products of AG codes, we need to analyze a similar operation in terms of the function field. That is, we need to understand the behaviour of spaces defined by products of elements of two finite dimensional subspaces of a given function field. Let us first introduce another notation: let $\mathbb{A}$ be a commutative unitary algebra over a field $\mathbb{K}$. Given two subspaces $V, W$ of $\mathbb{A}$, in the same way as for the Schur product, the product of $V$ and $W$ is defined as:

$$
V \cdot W:=\operatorname{Span}_{\mathbb{K}}\{v w \mid v \in V, w \in W\} .
$$

We define inductively $L^{(1)}=L$ and $L^{(t+1)}=L^{(t)} \cdot L$.

Theorem 5 ([31, Theorem 6]). Let E, F be two divisors on the curve $\mathcal{X}$ such that $\operatorname{deg}(E) \geqslant 2 g+1$ and $\operatorname{deg}(F) \geqslant 2 g$ and let $t$ be a positive integer. Then,

(1) $L(E) \cdot L(F)=L(E+F)$;

(2) $L(E)^{(t)}=L(t E)$.

Since the evaluation map is a morphism of algebras we deduce directly from Theorem 5 the following statement.

Corollary 6. Let $E, F$ be two divisors on the curve $\mathcal{X}$ both with disjoint support with $\mathcal{P}$ and such that $\operatorname{deg}(E) \geqslant 2 g+1$ and $\operatorname{deg}(F) \geqslant 2 g$ and let $t$ be a positive integer. Then,

(1) $\mathcal{C}_{L}(\mathcal{X}, \mathcal{P}, E) * \mathcal{C}_{L}(\mathcal{X}, \mathcal{P}, F)=\mathcal{C}_{L}(\mathcal{X}, \mathcal{P}, E+F)$

(2) $\mathcal{C}_{L}(\mathcal{X}, \mathcal{P}, E)^{(t)}=\mathcal{C}_{L}(\mathcal{X}, \mathcal{P}, t E)$

We conclude this subsection with the following statement which is crucial in the sequel.

Proposition 7. Let $E, F$ be two divisors on the curve $\mathcal{X}$ both with disjoint supports with $\mathcal{P}$ and such that $\operatorname{deg}(F) \geqslant 2 g$ and $\operatorname{deg}(E) \leqslant n-3$. Then,

$$
\begin{aligned}
\mathcal{C}_{L}(\mathcal{X}, \mathcal{P}, E-F) & =\left(\mathcal{C}_{L}(\mathcal{X}, \mathcal{P}, F) * \mathcal{C}_{L}(\mathcal{X}, \mathcal{P}, E)^{\perp}\right)^{\perp} \\
& =\left\{\mathbf{z} \in \mathbb{F}_{q}^{n} \mid \mathbf{z} * \mathcal{C}_{L}(\mathcal{X}, \mathcal{P}, F) \subseteq \mathcal{C}_{L}(\mathcal{X}, \mathcal{P}, E)\right\}
\end{aligned}
$$

Proof. Let $A=\mathcal{C}_{L}(\mathcal{X}, \mathcal{P}, F)$ and $B=\mathcal{C}_{L}(\mathcal{X}, \mathcal{P}, E)$. Theorem 3 shows that $B^{\perp}=\mathcal{C}_{L}\left(\mathcal{X}, \mathcal{P}, E^{\perp}\right)$ with $E^{\perp}=K-E+D_{\mathcal{P}}$ for some canonical divisor $K$. Next, since $\operatorname{deg}(E) \leqslant n-3$, we have $\operatorname{deg}\left(E^{\perp}\right) \geqslant 2 g+1$. Now,

$$
A * B^{\perp}=\mathcal{C}_{L}\left(\mathcal{X}, \mathcal{P}, D_{\mathcal{P}}+K-E+F\right)=\mathcal{C}_{L}(\mathcal{X}, \mathcal{P}, E-F)^{\perp},
$$

the first equality being a consequence of Corollary 6 and the last one due to Theorem 3 . The second equality of the statement follows from Lemma 4 


\subsection{Distinguisher and cryptanalysis}

Another and more recent application of the Schur product concerns cryptanalysis of code-based public key cryptosystems. In this context, the Schur product is a very powerful operation which can help to distinguish some algebraic codes such as AG codes from random ones. The point is that evaluation codes do not behave like random codes with respect to the Schur product: the square of an AG code is small compared to that of a random code of the same dimension. Thanks to this observation, Wieschebrink [46] gave an efficient attack of Berger Loidreau's proposal [1] based on subcodes of GRS codes.

Recent attacks consist in using this argument and take advantage of this distinguisher in order to compute a filtration of the public code by a family of very particular subcodes. This filtration method yields an alternative attack on GRS codes [6]. Next it lead to a key recovery attack on wild Goppa codes over quadratic extensions in [5, 10. Finally in the case of AG codes, this approach leads to an attack as we will see in Section 6. This attack consists in the computation of an error-correcting pair (ECP) or an error-correcting array (ECA) for the public code without retrieving the structure of the curve, the points and the divisor.

\section{The $s$-closure operation}

In this section we introduce a new operation which we call the $s$-closure. This operation will be crucial in Section 4 to attack a McEliece scheme based on subcodes of AG codes. Roughly speaking, given a random subcode $C$ of an $\mathrm{AG}$ code $\mathcal{C}_{L}(\mathcal{X}, \mathcal{P}, E)$, if the codimension of $C$ in $\mathcal{C}_{L}(\mathcal{X}, \mathcal{P}, E)$ is small enough, then, with a high probability, the 2-closure of $C$ provides the code $\mathcal{C}_{L}(\mathcal{X}, \mathcal{P}, E)$.

Definition 4 ( $s$-closure). Let $\mathbb{A}$ be a commutative unitary algebra over a field $\mathbb{K}$ and suppose that we have a subspace $L$ of $\mathbb{A}$. Let $s \geqslant 2$ be an integer, the $s$-closure of $L$ is defined by

$$
\bar{L}^{s}=\left\{f \in \mathbb{A} \mid f \cdot L^{(s-1)} \subseteq L^{(s)}\right\} .
$$

The space $L$ is called $s$-closed if $\bar{L}^{s}=L$.

Remark 1. Special cases are discussed in the sequel where $\mathbb{K}=\mathbb{F}_{q}$ and either $\mathbb{A}$ is the field of rational functions on the curve $\mathcal{X}$ and $L$ is a subspace of a Riemann-Roch space $L(E)$ for a divisor $E$ on the curve, or $\mathbb{A}=\mathbb{F}_{q}^{n}$ and $L$ is a subspace of $\mathbb{F}_{q}^{n}$.

\subsection{General properties}

We list below some properties of the $s$-closure operation.

Proposition 8. Let $L, M$ and $N$ be subspaces of $\mathbb{A}$ and $s \geqslant 2$ be an integer, then:

(1) $\bar{L}^{s}$ is a vector space over $\mathbb{K}$.

(2) We have the following increasing sequence: $L \subseteq \bar{L}^{2} \subseteq \cdots \subseteq \bar{L}^{s} \subseteq \bar{L}^{s+1}$.

(3) Let $f \in \mathbb{A}$. Then $f \cdot \bar{L}^{s} \subseteq \overline{(f \cdot L)}^{s}$. Equality holds if $f$ is invertible in $\mathbb{A}$.

(4) Let $\phi: \mathbb{A} \rightarrow \mathbb{K}$ be a linear form and consider the non degenerate symmetric bilinear form over $\mathbb{A}$ defined as $\varphi(a, b):=\phi(a b)$. Then

$$
\bar{L}^{s}=\left(L^{(s-1)} \cdot L^{(s)^{\perp \varphi}}\right)^{\perp_{\varphi}} .
$$

This holds in particular when $\mathbb{A}=\mathbb{F}_{q}^{n}$ and $\varphi$ is the standard inner product. 
Proof. Statement (11) follows from the $\mathbb{K}$-bilinearity of the product. To prove (2), let $f \in \bar{L}^{s}$. Thus,

$$
f \cdot L^{(s-1)} \subseteq L^{(s)} \Longrightarrow f \cdot L^{(s)} \subseteq L^{(s+1)} .
$$

Therefore, $f \in \bar{L}^{s+1}$. To prove (3), let $g \in f \cdot \bar{L}^{s}$. Then, $g=f a$ for some $a \in \bar{L}^{s}$ and

$$
g \cdot(f \cdot L)^{(s-1)}=f^{s} \cdot a \cdot L^{(s-1)} \subseteq f^{s} \cdot L^{(s)}=(f \cdot L)^{(s)}
$$

Therefore $g \in \overline{f \cdot L}$. If moreover $f$ is invertible, then one can reverse the proof and the equality holds. Finally the proof of (4) is in the very same spirit as that of Lemma 4using the adjunction formula $\varphi(f g, h)=\phi(f g h)=\varphi(f, g h)$, which holds for all $f, g, h \in \mathbb{A}$.

Notice that one can have $L \subseteq M$ while $\bar{L}^{s} \nsubseteq \bar{M}^{s}$ as illustrated by the following example.

Example 1. Let $\mathbb{A}=\mathbb{K}[x]$ and $L:=\operatorname{Span}\left\{1, x+x^{2}, x^{3}, x^{4}\right\}$ and $M:=L \oplus \operatorname{Span}\left\{x^{9}\right\}$. A computation gives

$$
L^{(2)}=\mathbb{K}[x]_{\leqslant 8} \quad \text { and } \quad M^{(2)}=\mathbb{K}[x]_{\leqslant 8} \oplus x^{9} \cdot L \oplus \operatorname{Span}\left\{x^{18}\right\},
$$

where $\mathbb{K}[x]_{\leqslant 8}$ denotes the finite dimensional subspace of $\mathbb{K}[x]$ of polynomials of degree less than or equal to 8 . Next, one proves easily that $x \in \bar{L}^{2}$ while $x \notin \bar{M}^{2}$ since $x \cdot x^{9}=x^{10} \notin M^{(2)}$. Therefore, $\bar{L}^{2} \nsubseteq \bar{M}^{2}$.

On the other hand, we have the following lemma.

Lemma 9. Let $\mathbb{A}$ be the field of rational functions on the curve $\mathcal{X}$, let $M=L(E)$ for a divisor $E$ on $\mathcal{X}$ and $s \geqslant 2$ be an integer. If $L$ is a subspace of $M, L^{(s)}=M^{(s)}$ and $\bar{M}^{s}=M$, then $\bar{L}^{s}=M$.

Proof. Assume that $L \subseteq L(E)$. Let $E_{0}=\sum a_{i} P_{i}$ be divisor satisfying $L \subseteq L\left(E_{0}\right) \subseteq L(E)$ and minimal for this property. Then, for all $i$ there exists a function $g_{i} \in L$ such that $v_{P_{i}}\left(g_{i}\right)=-a_{i}$. Take $f \in \bar{L}^{s}$. By definition,

$$
f \cdot g_{i}^{s-1} \in f \cdot L^{(s-1)} \subseteq L^{(s)} \subseteq L\left(E_{0}\right)^{(s)} \subseteq L\left(s E_{0}\right) .
$$

So $v_{P_{i}}\left(f g_{i}^{s-1}\right)=v_{P_{i}}(f)-(s-1) a_{i} \geqslant-s a_{i}$. Or equivalently, $v_{P_{i}}(f) \geqslant-a_{i}$ for all $i$. Therefore, $(f) \geqslant-E_{0}$, that is $f \in L\left(E_{0}\right) \subseteq L(E)=M$, and $\bar{L}^{s} \subseteq M$ is proved.

Conversely, let $f \in M$, we have $f \cdot L^{(s-1)} \subseteq f \cdot M^{(s-1)} \subseteq M^{(s)}=L^{(s)}$ and hence $f \in \bar{L}^{s}$.

\subsection{Closures of Riemann Roch spaces and AG codes}

We will show that certain Riemann-Roch spaces of AG codes are $s$-closed. For this sake we first need the following lemma.

Lemma 10. Let $E, F$ be two divisors on the curve $\mathcal{X}$ of genus $g$ with $\operatorname{deg}(E) \geqslant 2 g$. Then,

$$
E \leqslant F \quad \text { if and only if } \quad L(E) \subseteq L(F) .
$$

Proof. The "only if" part is obvious. Conversely, let $L(E) \subseteq L(F)$ and suppose that $E \nless F$. If $E=\sum m_{P} P$ and $F=\sum n_{P} P$ with each $m_{P}, n_{P} \in \mathbb{Z}$, then, since $E \nless F$, there is a place $P_{0}$ such that $m_{P_{0}}>n_{P_{0}}$. As $\operatorname{deg}(E) \geqslant 2 g$, by Riemann-Roch Theorem, $\operatorname{dim}(L(E))=\operatorname{deg}(E)+1-g>g$. We distinguish two different cases:

- If $\operatorname{deg}\left(E-P_{0}\right)>2 g-2$, then $\operatorname{dim}\left(L\left(E-P_{0}\right)\right)=\operatorname{deg}\left(E-P_{0}\right)+1-g<\operatorname{dim}(L(E))$. 
- If $\operatorname{deg}\left(E-P_{0}\right) \leqslant 2 g-2$, then $\operatorname{dim}\left(L\left(E-P_{0}\right)\right) \leqslant \frac{1}{2} \operatorname{deg}\left(E-P_{0}\right)+1$, by Clifford's Theorem. So $\operatorname{dim}\left(L\left(E-P_{0}\right)\right) \leqslant g<\operatorname{dim}(L(E))$.

In both cases, $\operatorname{dim}\left(L\left(E-P_{0}\right)\right)<\operatorname{dim}(L(E))$. Hence, there exists a rational function $f \in$ $L(E) \backslash L\left(E-P_{0}\right)$. That means that $v_{P_{0}}(f)=-m_{P_{0}}<-n_{P_{0}}$. So $f \notin L(F)$, which contradicts our initial assumption.

Proposition 11. Let $E$ be a divisor on $\mathcal{X}$ with $\operatorname{deg}(E) \geqslant 2 g+1$ and $s \geqslant 2$ be an integer. Then, $\overline{L(E)}^{s}=L(E)$.

Proof. Inclusion $L(E) \subseteq \overline{L(E)}^{s}$ is obvious. Conversely, let $f \in \overline{L(E)}^{s}$. By definition, $f$. $L(E)^{(s-1)} \subseteq L(E)^{(s)}$. From Theorem [5 (2), this gives $f \cdot L((s-1) E) \subseteq L(s E)$. A simple computation shows that $f \cdot L((s-1) E)=L((s-1) E-(f))$. Therefore, we have the inclusion $L((s-1) E-(f)) \subseteq L(s E)$ and, thanks to Lemma 10, we get $(s-1) E-(f) \leqslant s E$, which entails $(f) \geqslant-E$ and hence $f \in L(E)$.

In terms of AG codes, this leads to:

Proposition 12. Let $E$ be a divisor on the curve $\mathcal{X}$ such that $2 g+1 \leqslant \operatorname{deg}(E) \leqslant \frac{n-2}{t}$. Let $s$ be an integer with $s \geqslant 2$. Then,

$$
{\overline{\mathcal{C}_{L}(\mathcal{X}, \mathcal{P}, E)}}^{s}=\mathcal{C}_{L}(\mathcal{X}, \mathcal{P}, E)
$$

Proof. Proposition 8(4) gives that

$$
{\overline{\mathcal{C}_{L}(\mathcal{X}, \mathcal{P}, E)}}^{s}=\left(\mathcal{C}_{L}(\mathcal{X}, \mathcal{P}, E)^{(s-1)} *\left(\mathcal{C}_{L}(\mathcal{X}, \mathcal{P}, E)^{(s)}\right)^{\perp}\right)^{\perp}
$$

Now, from Corollary 6(2) $), \mathcal{C}_{L}(\mathcal{X}, \mathcal{P}, E)^{(s)}=\mathcal{C}_{L}(\mathcal{X}, \mathcal{P}, s E)$. Moreover, by Theorem 3, we have $\mathcal{C}_{L}(\mathcal{X}, \mathcal{P}, s E)^{\perp}=\mathcal{C}_{L}\left(\mathcal{X}, \mathcal{P},(s E)^{\perp}\right)$ with $(s E)^{\perp}=D_{\mathcal{P}}-s E+K$ for some canonical divisor $K$ on $\mathcal{X}$. By assumption, $\operatorname{deg}(E) \leqslant \frac{n-2}{s}$ and hence

$$
\operatorname{deg}\left((s E)^{\perp}\right)=n-\operatorname{deg}(s E)+2 g-2 \geqslant 2 g .
$$

As $\operatorname{deg}(E) \geqslant 2 g+1$, then, thanks to Corollary 6(1) yields

$$
\mathcal{C}_{L}(\mathcal{X}, \mathcal{P},(s-1) E) * \mathcal{C}_{L}(\mathcal{X}, \mathcal{P}, s E)^{\perp}=\mathcal{C}_{L}\left(\mathcal{X}, \mathcal{P}, D_{P}-E+K\right)=\mathcal{C}_{L}(\mathcal{X}, \mathcal{P}, E)^{\perp} .
$$

\subsection{A conjecture}

In [46] Wieschebrink asserts, without proving it that, with high probability, the square of a low codimensional subcode $C$ of a $\operatorname{GRS}_{k}(\mathbf{a}, \mathbf{b})$ is a GRS code. More precisely, in general, $C^{(2)}=\mathrm{GRS}_{2 k-1}(\mathbf{a}, \mathbf{b} * \mathbf{b})$. Wieschebrink uses this observation to break Berger and Loidreau's proposal [1]. It is natural to ask whether this property extends to low codimensional subcodes of AG codes. Some experimental results encourage us to establish the following conjecture.

Conjecture 1. Let $C$ be a uniformly random subcode of $\mathcal{C}_{L}(\mathcal{X}, \mathcal{P}, E)$ of dimension $\ell$ such that

$$
2 g+1 \leqslant \operatorname{deg}(E) \leqslant \frac{n-1}{2} \quad \text { and } \quad 2 k+1-g \leqslant\left(\begin{array}{c}
\ell+1 \\
2
\end{array}\right)
$$

where $k=\operatorname{deg}(E)+1-g$ is the dimension of $\mathcal{C}_{L}(\mathcal{X}, \mathcal{P}, E)$. Then, the probability that $C^{(2)}$ is different from $\mathcal{C}_{L}(\mathcal{X}, \mathcal{P}, 2 E)$ tends to 0 when $k$ tends to infinity. 
We give a proof along the lines of [26, Remark 5] for the special case of subcodes of GRS codes. Note that the case of GRS codes is a bit different in terms of the probabilistic model since for a GRS code, for the length to tend to infinity, the size of the alphabet needs to tend to infinity too. Evidences for this conjecture are discussed further below.

Corollary 13. Assume that Conjecture 1 holds. Let $2 g+1 \leqslant \operatorname{deg}(E) \leqslant \frac{n-2}{2}$. Let $k:=$ $\operatorname{deg}(E)+1-g$ be the dimension of $\mathcal{C}_{L}(\mathcal{X}, \mathcal{P}, E)$, such that $2 k+1-g \leqslant\left(\begin{array}{c}\ell+1 \\ 2\end{array}\right)$ for some $\ell$. Then, the equality $\bar{C}^{2}=\mathcal{C}_{L}(\mathcal{X}, \mathcal{P}, E)$ holds for uniformly random $\ell$-dimensional subcodes $C$ of $\mathcal{C}_{L}(\mathcal{X}, \mathcal{P}, E)$ with a probability tending to 1 when $k$ tends to infinity.

Proof. Suppose Conjecture 1 holds. Let $C$ be an $l$-dimensional subcode of $\mathcal{C}_{L}(\mathcal{X}, \mathcal{P}, E)$. By Conjecture 1, we would have that $C^{(2)}=\mathcal{C}_{L}(\mathcal{X}, \mathcal{P}, E)^{(2)}$ with high probability. Moreover $\mathcal{C}_{L}(\mathcal{X}, \mathcal{P}, E)$ is 2-closed by Proposition 12. Thus, applying Lemma 9, we conclude that $\bar{C}^{2}=\mathcal{C}_{L}(\mathcal{X}, \mathcal{P}, E)$ which completes the proof.

\section{Experiments around this conjecture}

To test the validity of the conjecture, we performed experiments as follows:

- Generate a random smooth irreducible plane curve over $\mathbb{F}_{q}$ using Magma command RandomPlaneCurve;

- Choose a divisor on the curve by selecting some random rational points and places of higher degree and sum them up;

- Compute the corresponding code $C$;

- Choose a random subcode $C^{\prime}$ of dimension $\ell$ such that $\ell$ is the least integer satisfying $\left(\begin{array}{c}\ell+1 \\ 2\end{array}\right) \geqslant \operatorname{dim} C^{(2)}$

- Compare $C^{(2)}$ and $C^{\prime(2)}$.

Remark 2. Note that in terms of the dimension $\ell$ of the subcode, we tested only the critical case i.e. the minimal value of $\ell$. Clearly cases with larger $\ell$ 's can only be more successful.

Such a test has been performed on 100 random curves over fields $\mathbb{F}_{q}$ with $2<q<200$. These curve had genus $6<g<36$. For each curve, and for any $2 g+2 \leqslant m \leqslant \frac{n+g-2}{2}$ we chose a divisor $G$ of degree $m$ and tested 10000 random subcodes. Among these 10000 tests the codes $C^{\prime(2)}$ fails to equal $C^{(2)}$ at most $0.5 \%$ of the times. Moreover, for more than $90 \%$ of pairs (curve, divisor), the failure rate is $0 \%$.

\section{Decoding algorithms of algebraic geometry codes}

\subsection{Error-correcting pairs}

The notion of error-correcting pair (ECP) for a linear code was introduced by Pellikaan [33, 34] and independently by Kötter [22].

Generally, given a positive integer $t$, a $t$-ECP for a linear code $C \subseteq \mathbb{F}_{q}^{n}$ is a pair of linear codes $(A, B)$ in $\mathbb{F}_{q}^{n}$ satisfying $A * B \subseteq C^{\perp}$ together with several inequalities relating $t$ and the dimensions and (dual) minimum distances of $A, B, C$. In a formal manner:

Definition 5. Let $C$ be a linear code in $\mathbb{F}_{q}^{n}$. A pair $(A, B)$ of linear codes over $\mathbb{F}_{q}^{n}$ is called a t-error correcting pair (ECP) for $C$ if the following conditions hold: 
(E.1) $(A * B) \subseteq C^{\perp}$,

(E.2) $k(A)>t$
(E.3) $d\left(B^{\perp}\right)>t$

(E.4) $d(A)+d(C)>n$.

It is shown that a linear code in $\mathbb{F}_{q}^{n}$ with a $t$-error correcting pair has a decoding algorithm which corrects up to $t$ errors with complexity $O\left(n^{3}\right)$. ECPs provide a unifying point of view for several classical bounded distance decoding algorithms for algebraic and AG codes. See [25] for further details.

Theorem 14 ([34, Theorem 3.3]). Let $\mathcal{X}$ be a curve of genus $g$ and $E$ be a divisor on $\mathcal{X}$ such that $n>\operatorname{deg}(E)>3 g-1$. Let $d^{*}:=\operatorname{deg}(E)+2-2 g$ be the Goppa designed distance of $\mathcal{C}_{L}(\mathcal{X}, \mathcal{P}, E)^{\perp}$ and $t:=\left\lfloor\left(d^{*}-1-g\right) / 2\right\rfloor$. Let $F$ be any divisor on $\mathcal{X}$ with disjoint support with $D_{\mathcal{P}}$ and $\operatorname{deg}(F)=t+g$. Then, the pair of codes defined by

$$
A=\mathcal{C}_{L}(\mathcal{X}, \mathcal{P}, F) \quad \text { and } \quad B=\mathcal{C}_{L}(\mathcal{X}, \mathcal{P}, E-F)
$$

is a $t$-ECP for $\mathcal{C}_{L}(\mathcal{X}, \mathcal{P}, E)^{\perp}$. Under the above conditions, such a divisor $F$ always exists.

Corollary 15. Under the conditions stated above and assuming that $\operatorname{deg}(E) \leqslant n-3$ and $t \geqslant 1$. Then,

$$
A=(B * C)^{\perp} .
$$

Proof. Notice that $C=\mathcal{C}_{L}(\mathcal{X}, \mathcal{P}, E)^{\perp}$ and

$$
\begin{aligned}
\operatorname{deg}(E-F) & =\operatorname{deg}(E)-\operatorname{deg}(F)=\operatorname{deg}(E)-(t+g)=\operatorname{deg}(E)-2 t+t-g \\
& \geqslant \operatorname{deg}(E)-d^{*}+1+t=2 g+t-1 \geqslant 2 g
\end{aligned}
$$

We now apply Proposition 7 to obtain the desired result.

Remark 3. From a cryptanalytic point of view, the above corollary asserts that, it is sufficient to know the codes $\mathcal{C}_{L}(\mathcal{X}, \mathcal{P}, E)$ and $\mathcal{C}_{L}(\mathcal{X}, \mathcal{P}, E-F)$ in order to get a $t$-ECP for $\mathcal{C}_{L}(\mathcal{X}, \mathcal{P}, E)$. Roughly speaking: if you know $A$ and $C$, then you know $B$.

\subsection{Error-correcting arrays}

The notion of majority voting of unknown syndromes was initiated by Feng-Rao [14] for AG codes and by Feng-Tzeng [15] for cyclic codes. Duursma in [11,12] treated it as majority coset decoding.

The philosophy of these algorithms can roughly be summarized as follows. Suppose we have a code $C_{1}$ for which we need a decoding algorithm, and a subcode $C_{2}$ for which we have a decoding algorithm. Coset decoding is an algorithm which has as input a word $\mathbf{y}_{1}$ such that $\mathbf{y}_{1} \in \mathbf{e}+C_{1}$, and as output $\mathbf{y}_{2}$ such that $\mathbf{y}_{2} \in \mathbf{e}+C_{2}$. In the present article, the code $C_{2}$ will always be $\{0\}$ for which there exists an obvious decoding algorithm.

These algorithms have a purely linear algebraic description using the notion of error correcting arrays (ECA) [21, 23, 35] or that of well behaving sequences [16]. Take notice that error correcting arrays deal with spaces of functions. In particular, in the case of AG codes, arrays consist in infinite collections of Riemann Roch spaces, while well behaving sequences are defined directly from error correcting codes without involving any other external data.

In this article we chose to adopt a slightly different point of view mixing the concepts of 35 ] and [16]. For that purpose we introduce the notion of array of codes which is very similar to the notion of error correcting array but now defined only with codes, without involving the function fields and Riemann Roch spaces. Such array of codes is strongly related to an error correcting array in the sense of $[21,23,35]$. Moreover, since we work only with codes, we also use the 
notion of well behaving pair which is necessary for the definition of the designed distance and for the decoding algorithm. The choice of this mixed point of view is motivated by two facts. First, from the cryptanalytic point of view, it is interesting to show how to design a decoding algorithm from the single data of a generator matrix of a code. On the other hand, the operation we perform on codes arise from natural operations on Riemann Roch spaces. For this reason, even if we do not directly compute Riemann Roch spaces the language of arrays seemed more convenient than that of well behaving sequences to describe and explain our calculations.

Definition 6. An array of codes is a triple $(\mathcal{A}, \mathcal{B}, \mathcal{C})$ of sequences of linear codes $\mathcal{A}=\left(A_{i}\right)_{1 \leqslant i \leqslant n}$, $\mathcal{B}=\left(B_{i}\right)_{1 \leqslant i \leqslant n}$ and $\mathcal{C}=\left(C_{i}\right)_{1 \leqslant i \leqslant n}$ satisfying the following conditions for all $i \in\{1, \ldots, n\}$,

(A.1) $\operatorname{dim}\left(A_{i}\right)=i, \quad \operatorname{dim}\left(B_{i}\right)=i \quad$ and $\quad \operatorname{dim}\left(C_{i}\right)=n-i ;$

(A.2) $\quad A_{i} \subseteq A_{i+1}, \quad B_{i} \subseteq B_{i+1} \quad$ and $\quad C_{i} \supseteq C_{i+1}$;

(A.3) for all $r \in\{1, \ldots, n\}$, there exists a pair $(i, j)$ such that $A_{i} * B_{j} \subseteq C_{r}^{\perp}$ and $A_{i} * B_{j} \nsubseteq C_{r-1}^{\perp}$.

In addition we introduce the function $\hat{r}:\{1, \ldots, n\}^{2} \rightarrow\{1, \ldots, n\}$ defined as follows.

Definition 7. Let $(\mathcal{A}, \mathcal{B}, \mathcal{C})$ be an error correcting array. The function $\hat{r}$ is defined as

$$
\hat{r}(i, j):=\min \left\{r \in\{1, \ldots, n\} \mid A_{i} * B_{j} \subseteq C_{r}^{\perp}\right\} .
$$

Remark 4. Condition (A 3 ) of arrays of codes asserts that $\hat{r}$ is surjective.

Remark 5 . Note that $\hat{r}$ is increasing in both arguments but not necessarily strictly increasing. In particular if for some pair $(i, j) \neq(n, n)$ we have $\hat{r}(i, j)=n$ then, it is clear that $\hat{r}(k, l)=n$ for all $k \geqslant i$ and $l \geqslant j$.

Definition 8. A pair $(i, j) \in\{1, \ldots, n\}^{2}$ is said to be well behaving (WB in short) if

$$
\forall\left(i^{\prime}, j^{\prime}\right) \text { such that } i \leqslant i^{\prime}, j \leqslant j^{\prime} \text { and }(i, j) \neq\left(i^{\prime}, j^{\prime}\right), \hat{r}\left(i^{\prime}, j^{\prime}\right)<\hat{r}(i, j) \text {. }
$$

For all $r \in\{1, \ldots, n\}$, set

- $\hat{n}_{r}:=\mid\{(i, j) \mid 1 \leqslant i, j \leqslant n,(i, j)$ is WB and $\hat{r}(i, j)=r+1\} \mid$;

- $\hat{d}_{r}:=\min \left\{\hat{n}_{r^{\prime}} \mid r \leqslant r^{\prime} \leqslant n\right\}$.

We have the following result:

Theorem 16. For any array of codes $(\mathcal{A}, \mathcal{B}, \mathcal{C})$ we have

$$
\hat{d}_{r} \leqslant d\left(C_{r}\right), \text { for all } 1 \leqslant r \leqslant n .
$$

Proof. The proof is very similar to that of [21, Theorem 2.5].

Definition 9. An array of codes $(\mathcal{A}, \mathcal{B}, \mathcal{C})$ in $\mathbb{F}_{q}^{n}$ is said to be $t$-error correcting for a code $C$ in $\mathbb{F}_{q}^{n}$ if there exists an $s$ with $1 \leqslant s \leqslant n$ such that $C=C_{s}$ and $t \leqslant \frac{\hat{d}_{s}-1}{2}$.

Theorem 17. A linear code $C$ in $\mathbb{F}_{q}^{n}$ with a t-ECA has a decoding algorithm which corrects up to t errors with complexity $\mathcal{O}\left(n^{3}\right)$.

Proof. See [14] and [21, Theorem 2.9].

Remark 6. In the literature, for instance in [35] or [21, Remark 2.10], the definition of a $t$-error correcting array for a code $C$ is more general than that of this article. Indeed, usually, an ECA for a code $C$ is associated to a sequence of codes $\left(C_{i}\right)_{\ell \leqslant i \leqslant u}$ where $C=C_{r}$ for some $r \in[\ell, u]$ and the code $C_{\ell}$ is either zero or has a $t$-ECP. This condition is sufficient to get a decoding algorithm. Indeed, if we receive the word $\mathbf{y}=\mathbf{e}+\mathbf{c}$ with $\mathbf{c} \in C$ and $\mathbf{e}$ has weight less than or equal to $t$, then, by coset decoding, we obtain a vector $\mathbf{y}_{\ell}$ such that $\mathbf{y}_{\ell}=\mathbf{e}+\mathbf{c}_{\ell}$ for some $\mathbf{c}_{\ell} \in C_{\ell}$. Next, either $C_{\ell}=\{0\}$ and we get directly e or $C_{\ell}$ has a $t$-ECP which we can use to obtain e.

We chose to avoid such a general definition since it is useless for our purpose. 


\section{The array of interest in this article}

Let $E$ be a divisor on $\mathcal{X}$ and $P$ be a rational point of $\mathcal{X}$. Let $\left(\alpha_{i}\right)_{i \in \mathbb{N}}$ be the non gap sequence at $P$ and $\left(\beta_{i}\right)_{i \in \mathbb{N}}$ the $E$-non gap sequence at $P$ (see Section $[1.2$ for further details on Weierstrass gaps). We introduce the finite sequences $\left(\hat{\alpha}_{i}\right)_{1 \leqslant i \leqslant n}$ and $\left(\hat{\beta}_{i}\right)_{1 \leqslant i \leqslant n}$ defined as follows:

- for all $i \in\{1, \ldots, n\}, \hat{\alpha}_{i}$ is the least integer such that $\operatorname{dim} \mathcal{C}_{L}\left(\mathcal{X}, \mathcal{P}, \hat{\alpha}_{i} P\right)=i$;

- for all $i \in\{1, \ldots, n\}, \hat{\beta}_{i}$ is the least integer such that $\operatorname{dim} \mathcal{C}_{L}\left(\mathcal{X}, \mathcal{P}, E+\hat{\beta}_{i} P\right)=i$.

Remark 7. For indexes $i$ such that the evaluation map is injective, i.e. such that $L\left(\alpha_{i} P-D\right)=0$ (resp. $L\left(E+\beta_{i} P-D\right)=0$ ), we have $\hat{\alpha}_{i}=\alpha_{i}$ (resp. $\hat{\beta}_{i}=\beta_{i}$ ) while for larger dimensions, we need to consider the contribution of $(-D)$-non-gaps at $P$ (resp. $(E-D)$-non-gaps).

We define the triple $(\mathcal{A}, \mathcal{B}, \mathcal{C})$ of sequences of linear codes in $\mathbb{F}_{q}^{n}$ as:

- $\forall i \in\{1, \ldots, n\}, A_{i}:=\mathcal{C}_{L}\left(\mathcal{X}, \mathcal{P}, \hat{\alpha}_{i} P\right)$

- $\forall j \in\{1, \ldots, n\}, \quad B_{j}:=\mathcal{C}_{L}\left(\mathcal{X}, \mathcal{P}, E+\hat{\beta}_{j} P\right)$;

- $\forall r \in\{1, \ldots, n\}, C_{r}:=\mathcal{C}_{L}\left(\mathcal{X}, \mathcal{P}, E+\hat{\beta}_{r} P\right)^{\perp}=B_{r}^{\perp}$.

Proposition 18. The above defined triple $(\mathcal{A}, \mathcal{B}, \mathcal{C})$ is an array of codes.

Proof. Conditions (A, and (A 2 2 ) are direct consequences of the definition of the $\hat{\alpha}_{i}$ and $\hat{\beta}_{j}$ 's. Moreover, it is easy to see that $A_{1}$ is the repetition code i.e. the code spanned by $(1, \ldots, 1)$. Hence for all $1 \leqslant i \leqslant n$, we have $A_{1} * B_{n-i}=B_{n-i}=C_{i}^{\perp}$ which gives (A 3 ).

Theorem 19. Assume that $\operatorname{deg}(E)<n$. Let $d^{*}=\operatorname{deg}(E)+2-2 g$ be the Goppa designed distance of $\mathcal{C}_{L}(\mathcal{X}, \mathcal{P}, E)^{\perp}$ and set $t=\left\lfloor\left(d^{*}-1\right) / 2\right\rfloor$. Then, the above described triple $(\mathcal{A}, \mathcal{B}, \mathcal{C})$ is a t-ECA for $\mathcal{C}_{L}(\mathcal{X}, \mathcal{P}, E)^{\perp}$.

Proof. Clearly, $\mathcal{C}_{L}(\mathcal{X}, \mathcal{P}, E)^{\perp}$ is an element of the sequence $\left(C_{i}\right)_{1 \leqslant i \leqslant n}$. Let $k$ be the dimension of $\mathcal{C}_{L}(\mathcal{X}, \mathcal{P}, E)^{\perp}$. By definition, we have $\mathcal{C}_{L}(\mathcal{X}, \mathcal{P}, E)^{\perp}=C_{n-k}$ and $\hat{\beta}_{n-k}=0$. The only thing we need to prove is that $\hat{d}_{n-k} \geqslant d^{*}$. This can be proved in a very similar fashion as 21, Corollary 3.9] which is a direct consequence of [21, Theorem 3.8]. The cited proof involves the set of pairs $(i, j)$ such that

$$
\alpha_{i}+\beta_{j}=\beta_{n-k}
$$

If we prove that for any such pair $(i, j)$ we have $\alpha_{i}=\hat{\alpha}_{i}$ and $\beta_{j}=\hat{\beta}_{j}$, then the proof of [21, Theorem 3.8] will apply mutatis mutandis in our setting.

Let $(i, j)$ be a pair of positive integers satisfying (2). Since we always have $\alpha_{i} \geqslant 0$, there exists $\beta_{j} \leqslant \beta_{n-k}=0$. But, the assumption $\operatorname{deg}(E)<n$, entails that $\operatorname{deg}\left(E+\beta_{j} P-D\right)<0$ and hence $L\left(E-\beta_{j} P-D\right)=\{0\}$. Consequently, $\beta_{j}=\hat{\beta}_{j}$. On the other hand we always have $\beta_{j} \geqslant-\operatorname{deg}(E)$ and hence $\alpha_{i} \leqslant \operatorname{deg}(E)<n$ which entails $L\left(\alpha_{i} P-D\right)=\{0\}$ and gives that $\hat{\alpha}_{i}=\alpha_{i}$.

\section{The $P$-filtrations}

Let $P$ be one of the points of the $n$-tuple $\mathcal{P}$. Let $E$ be a divisor on $\mathcal{X}$ that has disjoint support from $\mathcal{P}$. Let $\mathcal{P}^{\prime}$ be the $(n-1)$-tuple obtained from $\mathcal{P}$ by deleting $P$. In the present section we give an efficient way to obtain a $t_{P}-\mathrm{ECP}$ and a $t_{A}$-ECA for the code $\mathcal{C}_{L}\left(\mathcal{X}, \mathcal{P}^{\prime}, E\right)^{\perp}$ with

$$
d^{*}=\operatorname{deg}(E)-2 g+2, \quad t_{P}=\left\lfloor\frac{d^{*}-g-1}{2}\right\rfloor \quad \text { and } \quad t_{A}=\left\lfloor\frac{d^{*}-1}{2}\right\rfloor .
$$


By this manner, given a received word $\mathbf{y}=\mathbf{c}+\mathbf{e}$ where $\mathbf{c} \in \mathcal{C}_{L}(\mathcal{X}, \mathcal{P}, E)^{\perp}$ and $\mathbf{e}$ has weight $\leqslant t_{P}$ (resp. $\leqslant t_{A}$ ), one can proceed to decoding as follows. Thanks to our ECP (resp. ECA), one decodes the word $\mathbf{y}$ punctured at position $P$, which yields $\mathbf{c}$ punctured at this position. Then retrieving $\mathbf{c}$ consists only in correcting an erasure at position $P$.

Remark 8. A method to deduce directly an ECP for the non punctured code is described in Appendix A.

From the knowledge of a generator matrix of $\mathcal{C}_{L}(\mathcal{X}, \mathcal{P}, E)^{\perp}$ we aim at computing the sequences of codes $\left(A_{i}\right)_{1 \leqslant i \leqslant n}$ and $\left(B_{j}\right)_{1 \leqslant j \leqslant n}$ introduced in Section 4.2. For the computational aspects it is convenient to introduce another pair of sequences very similar to $\left(A_{i}\right)_{i}$ and $\left(B_{i}\right)_{i}$ but with different indexes. Namely, we introduce the sequences $\left(U_{i}\right)_{i \in \mathbb{Z}}$ and $\left(V_{i}\right)_{i \in \mathbb{Z}}$ of codes of length $n-1$ defined as

$$
\forall i \in \mathbb{Z}, \quad U_{i}:=\mathcal{C}_{L}\left(\mathcal{X}, \mathcal{P}^{\prime}, i P\right) \quad \text { and } \quad V_{i}:=\mathcal{C}_{L}\left(\mathcal{X}, \mathcal{P}^{\prime}, E+i P\right) .
$$

These sequences are related to the $A_{i}$ 's and $B_{j}$ 's by the relation:

$$
\forall i \in\{1, \ldots, n\}, \quad A_{i}=U_{\hat{\alpha}_{i}} \quad \text { and } \quad B_{i}=V_{\hat{\beta}_{i}} .
$$

The reader will observe that, despite the introduction of new notation, this other way of indexing the sequences is more convenient for explicit computation and for understanding the behaviour of these codes with respect to the Schur product.

\subsection{Structure of the sequences $\left(U_{i}\right)_{i}$ and $\left(V_{i}\right)_{i}$}

First one observes that for all $i<0, U_{i}=\{0\}$. On the other hand for all $i \geqslant n+(2 g)-2$ one can prove that $U_{i}=\mathbb{F}_{q}^{n-1}$. Indeed, set

$$
D_{\mathcal{P}^{\prime}}:=\sum_{P \in \mathcal{P}^{\prime}} P
$$

Then, for all integer $i$, the evaluation map $\operatorname{ev}_{\mathcal{P}^{\prime}}$ induces an isomorphism between $U_{i}$ and $L(i P) / L\left(i P-D_{\mathcal{P}^{\prime}}\right)$ and Riemann Roch theorem asserts that $\operatorname{dim} L((n+2 g-2) P)=n-1+g$ and $\operatorname{dim} L\left((n+2 g-2) P-D_{\mathcal{P}^{\prime}}\right)=g$ which proves that $\operatorname{dim} U_{n+2 g-2}=n-1$ and hence $U_{n+2 g-2}=\mathbb{F}_{q}^{n-1}$.

Next, one can split the sequence $\left(U_{i}\right)_{0 \leqslant i \leqslant n+2 g-2}$ in three parts represented by the three following diagrams. A first part $\left(U_{i}\right)_{0 \leqslant i \leqslant 2 g-2}$ in which some consecutive terms may be equal because of gaps at $P$ :

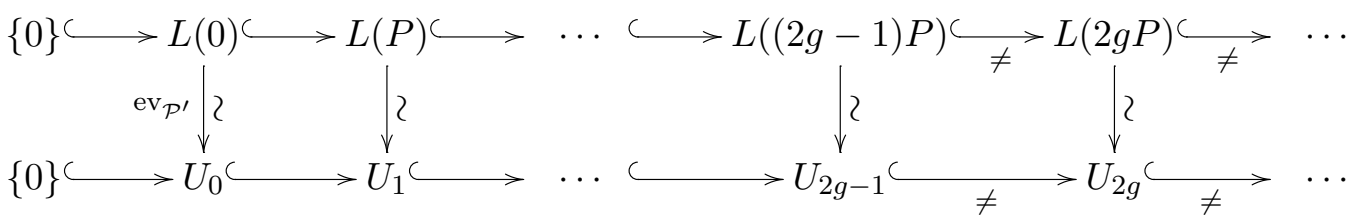

A second part $\left(U_{i}\right)_{2 g-1 \leqslant i \leqslant n-2}$ which is regular, i.e. any term has codimension 1 in the next one:

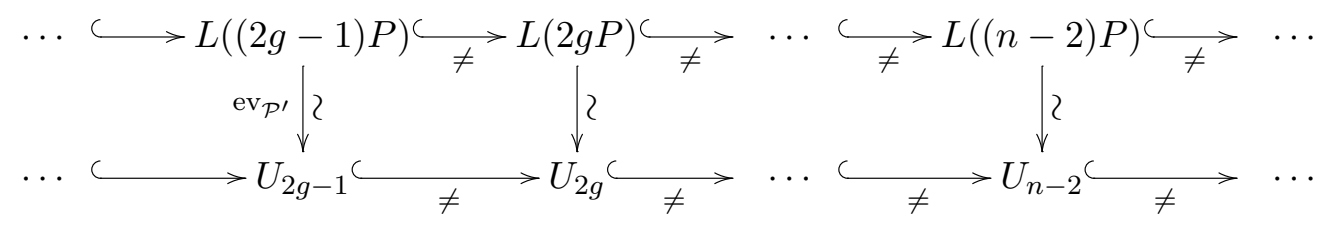


Finally, a third part $\left(U_{i}\right)_{n-1 \leqslant i \leqslant n+2 g-2}$ where the map ev $\mathcal{P}^{\prime}$ stops to be injective, but remains surjective. In this range, consecutive terms may be equal: it happens at every $\left(-D_{\mathcal{P}^{\prime}}\right)$-non-gap:

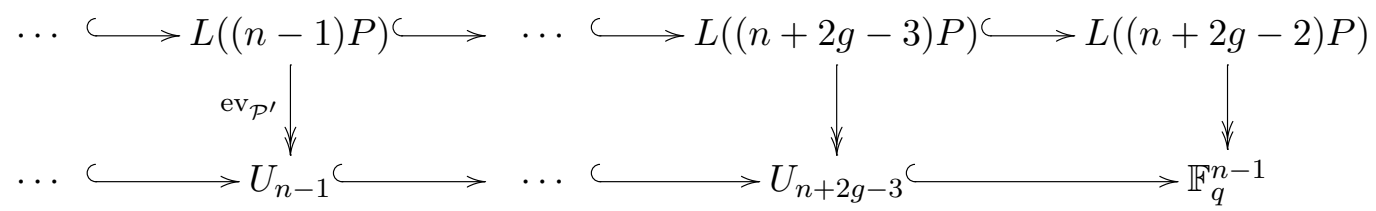

In the very same manner, for any $i<-\operatorname{deg}(E), V_{i}=\{0\}$ and for any $i \geqslant 2 g-2+n-\operatorname{deg}(E)$. Next, the sequence splits in three parts:

- A first part $\left(V_{i}\right)$ where $-\operatorname{deg}(E)+1 \leqslant i \leqslant-\operatorname{deg}(E)+2 g-1$ in which consecutive terms are equal at each $E$-gap at $P$;

- a second part $\left(V_{i}\right)$ where $-\operatorname{deg}(E)+2 g \leqslant i \leqslant-\operatorname{deg}(E)+n-2$ which is regular, i.e. any two consecutive terms are distinct;

- and a third part $\left(V_{i}\right)$ where $-\operatorname{deg}(E)+n-1 \leqslant i \leqslant-\operatorname{deg}(E)+n+2 g-2$ in which consecutive terms equal at each $\left(E-D_{\mathcal{P}^{\prime}}\right)$-non-gap.

\subsection{Effective computations}

Here we explain how to compute the terms of this sequence only by performing Schur products and solving linear systems. Notice that very similar methods have been used by Khuri-Makdisi to perform effective computations on Jacobians of curves [19].

\subsubsection{Which elements of the sequence do we know on the beginning?}

From a generator matrix of $\mathcal{C}_{L}(\mathcal{X}, \mathcal{P}, E)^{\perp}$, one can compute $\mathcal{C}_{L}(\mathcal{X}, \mathcal{P}, E)$. Then, $V_{0}$ and $V_{-1}$ are obtained from the code $\mathcal{C}_{L}(\mathcal{X}, \mathcal{P}, E)$ respectively by puncturing and shortening at the position $P$. All these operation boil down to Gaussian elimination.

\subsubsection{Computing terms of $\left(V_{i}\right)_{i}$ from other terms of $\left(V_{i}\right)_{i}$}

The following statement explains how to compute $V_{-i-1}$ (resp. $V_{i+1}$ ) from the knowledge of the codes $V_{-i}, V_{-i+1}$ (resp. $\left.V_{i}, V_{i-1}\right)$.

Proposition 20. Let $i \geqslant 1$, then we have

(i) if $\operatorname{deg}(E)-\frac{n-4}{2} \leqslant i \leqslant \operatorname{deg}(E)-2 g+1$, then

$$
V_{-i-1}=\left\{\mathbf{z} \in V_{-i} \mid \mathbf{z} * V_{-i+1} \subseteq V_{-i}^{(2)}\right\} ;
$$

(ii) if $\frac{n-4}{2}-\operatorname{deg}(E) \geqslant i \geqslant 2 g+1-\operatorname{deg}(E)$, then

$$
V_{i+1}=\left\{\mathbf{z} \in \mathbb{F}_{q}^{n-1} \mid \mathbf{z} * V_{i-1} \subseteq V_{i}^{(2)}\right\} .
$$

Proof. Assume that $\operatorname{deg}(E)-\frac{n-4}{2} \leqslant i \leqslant \operatorname{deg}(E)-2 g-1$. By Proposition 7 , the solution space of (3) is equal to

$$
V_{-i} \cap\left(V_{-i+1} *\left(V_{-i}^{(2)}\right)^{\perp}\right)^{\perp}
$$


Since $\operatorname{deg}(E-i P) \geqslant 2 g+1$, then, from Corollary 6, we have $V_{-i}^{(2)}=\mathcal{C}_{L}\left(\mathcal{X}, \mathcal{P}^{\prime}, 2 E-2 i P\right)$. Moreover, since $\operatorname{deg}(2 E-2 i P) \leqslant(n-1)-3$, from Proposition 7 we conclude that

$$
\left(V_{-i+1} *\left(V_{-i}^{(2)}\right)^{\perp}\right)^{\perp}=\mathcal{C}_{L}\left(\mathcal{X}, \mathcal{P}^{\prime},(2 E-2 i P)-(E-(i-1) P)\right)=V_{-i-1}
$$

This proves (ii). The proof of (iii) is very similar.

Therefore, we can define an algorithm for determining the code $V_{-i}$ for $i \geqslant 1$ wich consists in $i$ repeated applications of Proposition 20. But we can do better by decreasing the number of iterations and relaxing the parameters conditions using the following generalization of Proposition 20 whose proof is very similar.

Proposition 21. Let $a \leqslant b \leqslant c \leqslant d$ be integers such that $a+d=b+c$. If $\operatorname{deg}(E)+b \geqslant 2 g+1$ and $2 \operatorname{deg}(E)+b+c \leqslant n-4$ then,

(i) $V_{a}=\left\{\mathbf{z} \in V_{b} \mid \mathbf{z} * V_{d} \subseteq V_{b} * V_{c}\right\}$.

(ii) If moreover $\operatorname{deg}(E)+a \geqslant 2 g$, then $V_{d}=\left\{\mathbf{z} \in \mathbb{F}_{q}^{n-1} \mid \mathbf{z} * V_{a} \subseteq V_{b} * V_{c}\right\}$;

Remark 9. The previous statement permits for instance to compute $V_{-i}$ as

$$
V_{-i}=\left\{\mathbf{z} \in V_{\left\lfloor\frac{-i-1}{2}\right\rfloor} \mid \mathbf{z} * V_{0} \subseteq V_{\left\lfloor\frac{-i-1}{2}\right\rfloor} * V_{\left\lfloor-\frac{-i+1}{2}\right\rfloor}\right\} .
$$

In the same spirit as the quick exponentiation algorithm, the recursive application of the above formula allows to compute $V_{-i}$ in $O(\log (i))$ iterations of Proposition 21]instead of $O(i)$ iterations of Proposition 20.

\subsubsection{Further computations}

In the same manner if we know some terms of the sequence $\left(U_{i}\right)_{i}$ we can compute other ones as follows.

Proposition 22. Let $i, j$ be integers.

(i) If $i \geqslant 2 g$ and $j \geqslant 2 g+1$ then $U_{i} * U_{j}=U_{i+j}$.

(ii) Let $\ell$ be an integer such that $i+\ell=2 j$. If $i \geqslant 2 g$ and $2 g+1 \leqslant j \leqslant \frac{n-4}{2}$, then

$$
U_{\ell}=\left\{\mathbf{z} \in \mathbb{F}_{q}^{n-1} \mid \mathbf{z} * U_{i} \subseteq U_{j}^{(2)}\right\}
$$

It is also possible to compute some terms of one of the sequence from the knowledge of terms of the other sequence:

Proposition 23. Let $i, j, \ell$ such that $i+j=\ell, \operatorname{deg}(E)+\ell \leqslant n-4$. Then,

(i) If $\operatorname{deg}(E)+j \geqslant 2 g$, then $U_{i}=\left\{\mathbf{z} \in \mathbb{F}_{q}^{n-1} \mid \mathbf{z} * V_{j} \subseteq V_{\ell}\right\}$;

(ii) If $j \geqslant 2 g$, then $V_{i}=\left\{\mathbf{z} \in \mathbb{F}_{q}^{n-1} \mid \mathbf{z} * U_{j} \subseteq V_{\ell}\right\}$.

Finally, notice that some terms of $\left(V_{i}\right)_{i}$ can be constructed using the following statement.

Proposition 24. For all $i \geqslant 2 g$ and $j \geqslant 2 g-\operatorname{deg}(E)$ with either $i>2 g$ or $j>2 g-\operatorname{deg}(E)$, we have $V_{i+j}=U_{i} * V_{j}$. 


\subsubsection{Complexity}

The computation of one of the $U_{i}$ 's or $V_{i}$ 's using one of the previous statement consists in computing a finite number of Schur products. The cost of the computation of a Schur product is $O\left(n^{4}\right)$ (see for instance [6, Proposition 5]). A probabilistic shortcut allows to reduce the complexity of this computation to $O\left(n^{3+\varepsilon}\right)$ operation for $\varepsilon>0$ arbitrarily small. Indeed, given a code $C$ of length $n$ and dimension $k$, the computation of $C^{(2)}$ consists in computing the $\left(\begin{array}{c}k+1 \\ 2\end{array}\right)=O\left(n^{2}\right)$ generators and then, to perform Gaussian elimination to deduce a basis from this family of generators which costs $O\left(n^{4}\right)$. However, by extracting $n+\varepsilon$ elements chosen at random from this set of generators, we get another generating set with a large probability and this probabilistic trick reduces the complexity to $O\left(n^{3+\varepsilon}\right)$. A similar probabilistic shortcut is used in [20].

\section{The Attack}

\subsection{The McEliece encryption scheme}

Let $\mathcal{F}$ be a family of linear codes with an efficient decoding algorithm. Every element of this family is represented by the triple $\left(C, \mathcal{A}_{C}, t\right)$ where $\mathcal{A}_{C}$ denotes a decoding algorithm for $C \in \mathcal{F}$ which corrects up to $t$ errors. The McEliece scheme can be summarized as follows: Alice applies an encoding mechanism to a message and adds enough errors to make it unintelligible. Then, Bob is the only person that knows an efficient decoding method (the secret key) to detect and correct those errors. That is:

Key generation: Consider any element $\left(C, \mathcal{A}_{C}, t\right) \in \mathcal{F}$. Let $G$ be a generator matrix of $C$. Then the public key and the private key are given respectively by

$$
\mathcal{K}_{\text {pub }}=(G, t) \quad \text { and } \quad \mathcal{K}_{\text {secret }}=\mathcal{A}_{C} .
$$

Encryption: The plaintext $\mathbf{m}$ is encrypted as $\mathbf{y}=\mathbf{m} G+\mathbf{e}$ where $\mathbf{e}$ is a random error vector of weight at most $t$.

Decryption: Using $\mathcal{K}_{\text {secret }}$, the receiver obtains $\mathbf{m}$.

\subsection{Context of the present article}

In what follows, $\mathcal{X}$ denotes a smooth projective geometrically connected curve over $\mathbb{F}_{q}$ of genus $g, \mathcal{P}=\left(P_{1}, \ldots, P_{n}\right)$ denotes an $n$-tuple of mutually distinct $\mathbb{F}_{q}$-rational points of $\mathcal{X}, D_{\mathcal{P}}$ denotes the divisor $D_{\mathcal{P}}:=P_{1}+\cdots+P_{n}$ and $E$ denotes a divisor with disjoint support from that of $D_{\mathcal{P}}$.

We assume that our public key is a generator matrix $\mathbf{G}$ of the public code $\mathcal{C}_{L}(\mathcal{X}, \mathcal{P}, E)^{\perp}$ and the largest number $t$ of errors introduced during the encryption step. We take $t \leqslant\left\lfloor\left(d^{*}-1\right) / 2\right\rfloor$ where $d^{*}=\operatorname{deg}(E)-2 g+2$ is called the designed minimum distance of the public code $\mathcal{C}_{L}(\mathcal{X}, \mathcal{P}, E)^{\perp}$. Thus,

$$
C_{\text {pub }}: \text { : } \mathbf{G} \text { a generator matrix of } \mathcal{C}_{L}(\mathcal{X}, \mathcal{P}, E)^{\perp} \text { and } t .
$$

Our attack consists in the computation either of an error-correcting pair (ECP) or of an errorcorrecting array $(\mathrm{ECA})$ in order to decode $\mathcal{C}_{L}(\mathcal{X}, \mathcal{P}, E)^{\perp}$. For this sake, we distinguish two different cases:

$$
t_{P} \leqslant \frac{d^{*}-g-1}{2} \text { (i.e. related to ECP) and } t_{A} \leqslant \frac{d^{*}-1}{2} \text { (i.e. related to ECA). }
$$

Take notice that, from the single knowledge of a generator matrix of $\mathcal{C}_{L}(\mathcal{X}, \mathcal{P}, E)$, one can compute $\operatorname{deg}(E)$ and the genus $g$ of $\mathcal{X}$ using the following statement. 
Proposition 25 ([27, Proposition 18]). If $2 g+1 \leqslant \operatorname{deg}(E)<\frac{n}{2}$. Let $k_{1}$ and $k_{2}$ be the dimension of $\mathcal{C}=\mathcal{C}_{L}(\mathcal{X}, \mathcal{P}, E)$ and $\mathcal{C}^{(2)}$, respectively. Then,

$$
\operatorname{deg}(E)=k_{2}-k_{1} \quad \text { and } \quad g=k_{2}-2 k_{1}+1 .
$$

\subsection{In case $t \leqslant \frac{d^{*}-g-1}{2}$, i.e. computing an ECP}

In this section we describe how to attack the McEliece cryptosystem based on AG codes when $t \leqslant \frac{d^{*}-g-1}{2}$. If $\frac{n}{2}-2 \geqslant \operatorname{deg}(E) \geqslant 3 g+t-2$, then the attack summarizes as follows. The upper bound on $\operatorname{deg}(E)$ can be relaxed by applying techniques from Section 6.5. On the other hand, if $\operatorname{deg}(E)$ is below the lower bound $3 g+t-2$, one can still compute an ECA using the techniques of Section 6.4, which provides a more efficient decoding algorithm.

Remark 10. Note that for $t$ to be positive, the degree of $E$ should satisfy

$$
\operatorname{deg}(E) \geqslant 3 g .
$$

Indeed, we have $t \leqslant \frac{d^{*}-g-1}{2}=\frac{\operatorname{deg}(E)-3 g+1}{2}$.

Step 1. Determine the values $g$ and $\operatorname{deg}(E)$ using Proposition 25.

Step 2. Compute $V_{0}=\mathcal{C}_{L}\left(\mathcal{X}, \mathcal{P}^{\prime}, E\right)$ and $V_{-1}=\mathcal{C}_{L}\left(\mathcal{X}, \mathcal{P}^{\prime}, E-P\right)$ by Gaussian elimination.

Step 3. Compute the code $V_{-t-g}=\mathcal{C}_{L}\left(\mathcal{X}, \mathcal{P}^{\prime}, E-(t+g) P\right)$ using Proposition 20(1) or Remark 9 .

Step 4. Apply Corollary 15 to deduce an ECP for $\mathcal{C}_{\text {pub }}$ (punctured at the position $P$ ).

Remark 11. Remind that the above procedure provides an ECP for the code $\mathcal{C}_{L}(\mathcal{X}, \mathcal{P}, E)^{\perp}$ punctured at one position. But as explained in Section [5, the decoding of $\mathcal{C}_{L}(\mathcal{X}, \mathcal{P}, E)^{\perp}$ can be performed by first correcting errors on the punctured code and then correct an erasure on $\mathcal{C}_{L}(\mathcal{X}, \mathcal{P}, E)^{\perp}$. A method to get directly an ECP for $\mathcal{C}_{L}(\mathcal{X}, \mathcal{P}, E)^{\perp}$ is presented in Appendix A

Complexity The costly part of the procedure is the calculation of $V_{-t-g}$. If we proceed to $t+g$ iterations of Proposition 20(1i), then from Section 5.2.4, the complexity is $O\left((t+g) n^{4}\right)$. Using Remark 9 the cost can be reduced to $O\left(\log (t+g) n^{4}\right)$. Then, using the probabilistic shortcut explained in Section 5.2.4 we get a complexity in $O\left(\log (t+g) n^{3+\varepsilon}\right)$.

Experimental results Our attack has been implemented with MAGMA [3], we summarize in the following tables the average running times for several examples of codes, obtained with an Intel ${ }^{\circledR}$ CoreTM 2 Duo $2.8 \mathrm{GHz}$. The table includes for each code its base field size $q$, its length $n$, its dimension $k$, the correction capability $t$ when using error correcting pairs and the key size $\left\lceil(n-k) k \log _{2} q\right\rceil \cdot 10^{-3} \mathrm{kbits}$. The last column indicates the running time for the computation of an ECP for the public code. Moreover, the work factor $\mathbf{w}$ of and ISD attack is given. These work factors have been computed thanks to Christiane Peter's Software [37].

Example 2. The Hermitian curve $\mathcal{H}_{r}$ over $\mathbb{F}_{q}$ with $q=r^{2}$ is defined by the affine equation $Y^{r}+Y=X^{r+1}$. This curve has $P_{\infty}=(0: 1: 0)$ as the only point at infinity. Take $E=m P_{\infty}$

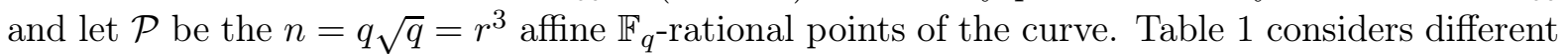
codes of type $C_{L}\left(\mathcal{H}_{r}, \mathcal{P}, E\right)^{\perp}$ with $n>m>2 g-2$.

Example 3. The Suzuki curves are curves $\mathcal{X}$ defined over $\mathbb{F}_{q}$ by the following equation $Y^{q}-Y=$ $X^{q_{0}}\left(X^{q}-X\right)$ with $q=2 q_{0}^{2} \geqslant 8$ and $q_{0}=2^{r}$ This curve has exactly $q^{2}+1$ rational places and a single place at infinity $P_{\infty}$. Let $E=m P_{\infty}$ and $\mathcal{P}$ be the $q^{2}$ rational points of the curve. Table 2 considers a code of type $\mathcal{C}_{L}(\mathcal{X}, \mathcal{P}, E)^{\perp}$ with $n>m>2 g-2$. 


\begin{tabular}{|c|c|c|c|c|c|c|c|}
\hline$q$ & $g$ & $n$ & $k$ & $t$ & $\mathbf{w}$ & key size & time \\
\hline \hline $7^{2}$ & 21 & 343 & 193 & 54 & $2^{84}$ & $163 \mathrm{kbits}$ & $74 \mathrm{~s}$ \\
\hline $9^{2}$ & 36 & 729 & 404 & 126 & $2^{182}$ & $833 \mathrm{kbits}$ & $21 \mathrm{~min}$ \\
\hline $11^{2}$ & 55 & 1331 & 885 & 168 & $2^{311}$ & $2730 \mathrm{kbits}$ & $67 \mathrm{~min}$ \\
\hline
\end{tabular}

Table 1: Comparison with Hermitian codes

\begin{tabular}{|c|c|c|c|c|c|c|c|}
\hline$q$ & $g$ & $n$ & $k$ & $t$ & $\mathbf{w}$ & key size & time \\
\hline \hline $2^{5}$ & 124 & 1024 & 647 & 64 & $2^{110}$ & $1220 \mathrm{kbits}$ & $30 \mathrm{~min}$ \\
\hline
\end{tabular}

Table 2: Comparison with Suzuki codes

\subsection{In case $t \leqslant \frac{d^{*}-1}{2}$, i.e. computing an ECA}

In this section we describe how to attack the McEliece cryptosystem based on AG codes when the integer $t$ is smaller than $\frac{d^{*}-1}{2}$. We first suppose that $4 g-1 \leqslant \operatorname{deg}(E) \leqslant \frac{n-4}{2}$. The lower degree case will be treated further. Then, one can compute the sequences $\left(U_{i}\right)_{i}$ and $\left(V_{i}\right)_{i}$ as follows.

Step 0 As explained in Section 5.2.1, compute $V_{0}$ and $V_{-1}$.

Step 1 By applying iteratively Proposition 20(1) on can compute $V_{-2}, \ldots, V_{-2 g-1}$ from the knowledge of $V_{0}, V_{-1}$. These computations are possible under the above conditions on $\operatorname{deg}(E)$.

Step 2 Compute $U_{0}, \ldots, U_{2 g+1}$ using Proposition 23(ii).

Step 3 Compute $U_{2 g+2}, \ldots, U_{4 g+2}$ using Proposition 22(1ii), then compute the rest of the sequence $\left(U_{i}\right)_{i}$ using Proposition 22)(ii).

Step 4 Compute the $V_{i}$ 's for $i \geqslant 0$ using Proposition 24.

Step 5 Compute the remaining $V_{i}$ 's (for $i<-2 g-1$ ) using either Proposition 23)(ii) or Proposition 21(1).

Assume now that $2 g+1 \leqslant \operatorname{deg}(E) \leqslant 4 g+1$, one can proceed as follows. First, using Corollary [6 , one can compute $\mathcal{C}_{L}\left(\mathcal{X}, \mathcal{P}^{\prime}, 2 E\right)$ as $\mathcal{C}_{L}\left(\mathcal{X}, \mathcal{P}^{\prime}, E\right)^{(2)}$. Next, since $\operatorname{deg}(2 E) \geqslant 4 g+1$, using the above described process, one can compute the whole filtrations

$$
U_{i}=\mathcal{C}_{L}\left(\mathcal{X}, \mathcal{P}^{\prime}, i P\right) \quad \text { and } \quad V_{i}^{\prime}=\mathcal{C}_{L}\left(\mathcal{X}, \mathcal{P}^{\prime}, 2 E+i P\right) .
$$

If $E \geqslant 0$, then, one can compute any $V_{i}=\mathcal{C}_{L}\left(\mathcal{X}, \mathcal{P}^{\prime}, E+i P\right)$ such that $2 \operatorname{deg}(E)+i<n$ as

$$
\mathcal{C}_{L}\left(\mathcal{X}, \mathcal{P}^{\prime}, E+i P\right)=\mathcal{C}_{L}\left(\mathcal{X}, \mathcal{P}^{\prime}, E\right) \cap \mathcal{C}_{L}\left(\mathcal{X}, \mathcal{P}^{\prime}, 2 E+i P\right) .
$$

If $E \nsupseteq 0$, one can compute the $V_{i}$ 's for $i \geqslant 2 g$ using Proposition 24. Next, the $V_{i}$ 's for the other values of $i$ can be computed using iteratively Proposition 23(ii).

Remark 12. Note that the case $\operatorname{deg}(E) \leqslant 2 g$ is irrelevant. Indeed, since the Goppa designed distance is $\operatorname{deg}(E)+2-2 g$, considering codes with $\operatorname{deg}(E) \leqslant 2 g$ would mean that the Goppa designed distance is $\leqslant 2$ and hence no error can be corrected.

Complexity Since we have $O(n)$ codes to compute using methods described in Section 5.2.1. according to Section 5.2.4, the complexity of the construction of the sequences is $O\left(n^{5}\right)$ if we use a deterministic algorithm and $O\left(n^{4+\varepsilon}\right)$ if we use the probabilistic shortcut. 


\subsection{Extending the attack}

We have been working under the assumption that $\operatorname{deg}(E) \leqslant \frac{n}{2}-2$. In the remainder of this section we will see how this condition can be weakened.

\subsubsection{By dualizing}

A first manner to address the case $\operatorname{deg}(E)>\frac{n}{2}-2$ is to consider the dual code. Since, from Theorem 3, $\mathcal{C}_{L}\left(\mathcal{X}, \mathcal{P}^{\prime}, E\right)^{\perp}=\mathcal{C}_{L}\left(\mathcal{X}, \mathcal{P}^{\prime}, E^{\perp}\right)$ with $\operatorname{deg}\left(E^{\perp}\right)=n-\operatorname{deg}(E)+2 g-2$. Next suppose that $\operatorname{deg}\left(E^{\perp}\right) \leqslant \frac{n}{2}-2$, then, using the previous results one can compute the whole filtrations

$$
U_{i}=\mathcal{C}_{L}\left(\mathcal{X}, \mathcal{P}^{\prime}, i P\right) \quad \text { and } \quad W_{i}=\mathcal{C}_{L}\left(X, P^{\prime}, E^{\perp}+i P\right)
$$

and there remains to notice that

$$
W_{i}^{\perp}=\mathcal{C}_{L}\left(\mathcal{X}, \mathcal{P}^{\prime}, E-i P\right)=V_{-i},
$$

which permits to compute the filtration $\left(V_{i}\right)_{i}$.

In summary, this dualization approach permits to treat the case $\operatorname{deg}(E) \geqslant \frac{n}{2}+2 g$. There remains to treat the case where

$$
\frac{n}{2}-2<\operatorname{deg}(E)<\frac{n}{2}+2 g .
$$

This issue is partially addressed in the next section.

\subsubsection{By shortening}

Up to now, we explained how to break the system unless:

$$
\frac{n}{2}-2<\operatorname{deg}(E)<\frac{n}{2}+2 g .
$$

Note that, according to the previous section, one can either work on the code or its dual. Therefore, there remains to treat the case

$$
\frac{n}{2}-2<\operatorname{deg}(E)<\frac{n}{2}+g .
$$

Notation 1. Consider the code $C=\mathcal{C}_{L}(\mathcal{X}, \mathcal{P}, E)$. Let $I$ be a subset of $\{1, \ldots, n\}$ and $P_{I}$ the divisor $\sum_{j \in I} P_{j}$. The code $\mathcal{C}_{L}\left(\mathcal{X}, \mathcal{P}, E-P_{I}\right)$ is denoted by $C(I)$.

If we delete the zero positions of the code $C(I)$ we obtain a code of length $n-|I|$ which is nothing but the shortening of $C$ at $I$. The interest of shortening is that

$$
|I| \geqslant 2 \operatorname{deg}(E)-n+4 \Longrightarrow \operatorname{deg}\left(E-P_{I}\right)<\frac{n-|I|}{2} .
$$

Hence, if $\operatorname{deg}(E) \geqslant \frac{n}{2}-2$, then for a set of indexes $I$ such that $|I|$ is large enough, the shortened code at $I$ satisfies the degree constraint.

This can be applied for cryptanalysis as follows. For instance, suppose we know $V_{i-1}$ and $V_{i}$ and aim to compute $V_{i+1}$ using Proposition 20(iii) but unfortunately $\operatorname{deg}(E)+i P \geqslant \frac{n}{2}-2$. Then, choose some sets of indexes $I_{1}, \ldots, I_{s}$ satisfying (5) and compute the codes $V_{i}\left(I_{1}\right), \ldots, V_{i}\left(I_{s}\right)$, and $V_{i-1}\left(I_{1}\right), \ldots, V_{i-1}\left(I_{s}\right)$ by Gaussian elimination. Afterwards, using Proposition 20(ii), deduce from them the codes $V_{i+1}\left(I_{1}\right), \ldots, V_{i+1}\left(I_{s}\right)$ and sum them up. The following statement asserts that this sum of codes equals $V_{i+1}$ provided some mild conditions on the $I_{j}$ 's are satisfied. 
Lemma 26. Let $F$ be a divisor of degree less than $n$. Let $I_{1}, \ldots, I_{s}$ be subsets of $\{1, \ldots, n\}$ such that $\operatorname{deg}(F)-\left|\bigcup_{j=1}^{s} I_{j}\right|>2 g-2$. Then,

$$
C\left(I_{1} \cap \cdots \cap I_{s}\right)=C\left(I_{1}\right)+\cdots+C\left(I_{s}\right) .
$$

In particular, if $I_{1} \cap \cdots \cap I_{s}=\emptyset$, then $C=C\left(I_{1}\right)+\cdots+C\left(I_{s}\right)$.

Proof. Since $\operatorname{deg}(F)<n$, the evaluation map is injective on $L(F)$. Hence it is sufficient to prove that

$$
L\left(F-P_{I_{1} \cap \cdots \cap I_{s}}\right)=L\left(F-P_{I_{1}}\right)+\cdots+L\left(F-P_{I_{s}}\right) .
$$

We give the proof for the case $s=2$. The general case deduces from that one using a descending induction on $s$. First, since $F-P_{I_{1}} \leqslant F-P_{I_{1} \cap I_{2}}$ and $F-P_{I_{2}} \leqslant F-P_{I_{1} \cap I_{2}}$ we get

$$
L\left(F-P_{I_{1}}\right)+L\left(F-P_{I_{1}}\right) \subseteq L\left(F-P_{I_{1} \cap I_{2}}\right) .
$$

Conversely, we will prove that both sides have the same dimension. For this sake one first observes that

$$
L\left(F-P_{I_{1}}\right) \cap L\left(F-P_{I_{2}}\right)=L\left(F-P_{I_{1} \cup I_{2}}\right),
$$

which entails that

$$
\begin{aligned}
\operatorname{dim}\left(L\left(F-P_{I_{1}}\right)+L\left(F-P_{I_{2}}\right)\right) & = \\
& \operatorname{dim}\left(L\left(F-P_{I_{1}}\right)\right)+\operatorname{dim}\left(L\left(F-P_{I_{2}}\right)\right)-\operatorname{dim}\left(L\left(F-P_{I_{1} \cup I_{2}}\right)\right) .
\end{aligned}
$$

By assumption on $\left|I_{1} \cup I_{2}\right|$, all the degrees of the above involved divisors exceed $2 g-2$ and hence, from Riemann Roch theorem,

$$
\operatorname{dim}\left(L\left(F-P_{I_{1}}\right)+L\left(F-P_{I_{2}}\right)\right)=\operatorname{deg}(F)-g+1-\left(\left|I_{1}\right|+\left|I_{2}\right|-\left|I_{1} \cup I_{2}\right|\right),
$$

which is nothing but $\operatorname{dim} L\left(F-P_{I_{1} \cap I_{2}}\right)$. This concludes the proof.

If we go back to the situation

$$
\frac{n}{2}-2<\operatorname{deg}(E)<\frac{n}{2}+g .
$$

We have $\operatorname{deg}(E)=\frac{n}{2}-2+\varepsilon$ for some $1 \leqslant \varepsilon \leqslant g-1$. Therefore, we need to shorten the code at least at $2 \varepsilon$ positions. Moreover, for the attack to work on the shortened code, we need $\operatorname{deg}(E)-2 \varepsilon>2 g$. In the worst case, $\varepsilon=g-1$ and the attack on the shortened code is proved to be efficient only if $\operatorname{deg}(E)>4 g-2$.

\subsubsection{Are there codes out of the reach of the attack?}

For the codes such that

$$
\frac{n}{2}-2<\operatorname{deg}(E)<\frac{n}{2}+g
$$

and such that $\operatorname{deg}(E) \leqslant 4 g-2$, the previously described attack is not proved to be efficient. Such codes can be obtained from Garcia Stichtenoth towers by taking a curve with a large genus $g$ and whose number of points is $\approx c \cdot g$ for some positive constant $c$. In this context, one can construct a code of length $n=c^{\prime} \cdot g$ for some positive constant $c^{\prime}<c$ and consider a divisor $E$ of degree $\frac{n}{2}+g$. If $c^{\prime}<6$ any of the previously described method cannot be proved to work.

However, for such codes, we can still try to apply the algorithms even if we have no proof they will provide the expected result. For a divisor $A$ of degree $<2 g+1$, the code $\mathcal{C}_{L}(\mathcal{X}, \mathcal{P}, A)^{(2)}$ may be non equal to the code $\mathcal{C}_{L}(\mathcal{X}, \mathcal{P}, 2 A)$ but is at least a subcode of it. This subcode can 
be used to try to compute subcodes of the form $\mathcal{C}_{L}\left(\mathcal{X}, \mathcal{P}^{\prime}, A-i P\right)$. Moreover, the previous approach using shortening leaves us many lattitude, since there is a large family of subsets we can use. Thus, by trying many tuples of subsets $\left(I_{1}, \ldots, I_{s}\right)$ we will probably be able to collect vectors of a target space $U_{i}$ or $V_{i}$ and after sufficiently many trials, get the whole target space. In addition that if this approach turned out to fail on both $\mathcal{C}_{L}\left(\mathcal{X}, \mathcal{P}^{\prime}, E\right)$ and $\mathcal{C}_{L}\left(\mathcal{X}, \mathcal{P}^{\prime}, E^{\perp}\right)$, it is always possible to choose another point $P$ in the support $\mathcal{P}$ and re-try with another $\mathcal{P}^{\prime}$.

Despite the lack of proofs in this situation, the existence of a code for which, the attack would fail for both $\mathcal{C}_{L}\left(\mathcal{X}, \mathcal{P}^{\prime}, E\right)$ and $\mathcal{C}_{L}\left(\mathcal{X}, \mathcal{P}^{\prime}, E^{\perp}\right)$, for any choice of shortening and for any choice of point $P \in \mathcal{P}$ seems highly improbable.

\section{Subcodes of AG codes}

In this section we give a polynomial time attack on the McEliece public key cryptosystem based on subcodes of AG codes. Now, our public key is a non structured generator matrix $\mathbf{G}$ of a subcode $C$ of $\mathcal{C}_{L}(\mathcal{X}, \mathcal{P}, E)^{\perp}$ of dimension $\ell$, together with the error correcting capacity $t$. Our attack consists in recovering the code $\mathcal{C}_{L}(\mathcal{X}, \mathcal{P}, E)^{\perp}$ from the knowledge of $C$ and then use one of the attacks presented in Section 6. That is, once $\mathcal{C}_{L}(\mathcal{X}, \mathcal{P}, E)^{\perp}$ is recovered, we design an efficient decoding algorithm for $\mathcal{C}_{L}(\mathcal{X}, \mathcal{P}, E)^{\perp}$ which corrects up to $t$ errors. This yields a decoding algorithm for our public code $C$.

The genus zero case (i.e. the case of GRS codes) proposed in [1] was broken by Wieschebrink [46] as follows:

- the public key $C$ is contained in some secret $\operatorname{GRS}_{k}(\mathbf{a}, \mathbf{b})$;

- compute $C^{(2)}$ which is, with a high probability, equal to $\operatorname{GRS}_{k}(\mathbf{a}, \mathbf{b})^{(2)}$, which is itself equal to $\mathrm{GRS}_{2 k-1}(\mathbf{a}, \mathbf{b} * \mathbf{b})$.

- Apply Sidelnikov Shestakov attack [42] to recover $\mathbf{a}$ and $\mathbf{b} * \mathbf{b}$, then recover $\mathbf{b}$.

Compared to Wieschebrink's approach, our difficulty is that our attack is not a key-recovery attack but a blind construction of a decoding algorithm. For this reason, even if $C^{(2)}$ provides probably the code $\mathcal{C}_{L}(\mathcal{X}, \mathcal{P}, E)^{(2)}$, it is insufficient for our purpose: we need to find $\mathcal{C}_{L}(\mathcal{X}, \mathcal{P}, E)$. This is the reason why we introduced the notion of $s$-closures in Section 3 .

\subsection{Principle of the attack}

In this section the public key consists in an $\ell$-dimensional subcode $C$ of the $\mathrm{AG} \operatorname{code} \mathcal{C}_{L}(\mathcal{X}, \mathcal{P}, E)^{\perp}$. Let $k:=\operatorname{dim} \mathcal{C}_{L}(\mathcal{X}, \mathcal{P}, E)$. We assume that

$$
2 g+1 \leqslant \operatorname{deg}(E) \leqslant \frac{n-1}{2} \quad \text { and } \quad 2 k-1+g \leqslant\left(\begin{array}{c}
l+1 \\
2
\end{array}\right) .
$$

Moreover, assume Conjecture 1 to be true. Then, with a high probability, we may assume that $C^{(2)}=\mathcal{C}_{L}(\mathcal{X}, \mathcal{P}, 2 E)$ and hence $\bar{C}^{2}=\mathcal{C}_{L}(\mathcal{X}, \mathcal{P}, E)$ by Corollary 13, Therefore, to break the scheme we can proceed as follows.

Step 1. Compute $\bar{C}^{2}$ by applying Proposition 7, which boils down to Gaussian elimination.

Step 2. Apply the polynomial time attack presented in Section 6 to obtain an ECP or an ECA for $\mathcal{C}_{L}(\mathcal{X}, \mathcal{P}, E)^{\perp}$. Which yields a decoding algorithm for $C$.

Remark 13. In case $\operatorname{deg}(E)>\frac{n-1}{2}$, then the attack can be applied to several shortenings of $C$ whose 2-closures are computed separately and are then summed up to provide $\mathcal{C}_{L}(\mathcal{X}, \mathcal{P}, E)$. This method is described in Section 6.5. 
Complexity: The computation of a closure costs $O\left(n^{4}\right)$ operations in $\mathbb{F}_{q}$. It can be reduced to $O\left(n^{3+\varepsilon}\right)$ operations using the probabilistic shortcut presented in Section 5.2.4. According to the previous results, the rest of the attack is at most in $O\left(n^{5}\right)$.

Experimental results This attack has been implemented with MAGMA. To this end $L$ random subcodes of dimension $\ell$ from Hermitian codes of parameters $[n, k]_{q}$ were created. It turned out that for all created subcodes a $t$-ECP could be reconstructed. Time represents the average time of the computation of an Error correcting pair for the public code obtained with an Intel $®$ CoreTM 2 Duo $2.8 \mathrm{GHz}$. The work factor $\mathbf{w}$ of an ISD attack is given. These work factors have been computed thanks to Christiane Peter's Software [37.

\begin{tabular}{|c|c|c|c|c|c|c|c|c|}
\hline$q$ & $n$ & $k$ & $t$ & Time & key size & w & $l$ & $L$ \\
\hline \hline & & & & & $83 \mathrm{kbits}$ & $2^{30}$ & 50 & 1000 \\
$7^{2}$ & \multirow{2}{*}{343} & \multirow{2}{*}{53} & \multirow{2}{*}{54} & $80 \mathrm{~s}$ & $137 \mathrm{kbits}$ & $2^{43}$ & 100 & 1000 \\
& & & & & $163 \mathrm{kbits}$ & $2^{62}$ & 150 & 1000 \\
\hline
\end{tabular}

\begin{tabular}{|c|c|c|c|c|c|c|c|c|}
\hline$q$ & $n$ & $k$ & $t$ & Time & key size & w & $l$ & $L$ \\
\hline \hline \multirow{2}{*}{$9^{2}$} & \multirow{2}{*}{729} & \multirow{2}{*}{521} & \multirow{2}{*}{19} & \multirow{3}{*}{$30 \mathrm{~min}$} & $216 \mathrm{ko}$ & $2^{32}$ & 50 & 500 \\
& & & & & $835 \mathrm{ko}$ & $2^{121}$ & 200 & 500 \\
& & & & & & \\
\end{tabular}

Table 3: Running times of the attack over Hermitian codes

\subsection{Which codes are subject to this attack?}

The subcode $C \subseteq \mathcal{C}_{L}(\mathcal{X}, \mathcal{P}, E)$ should satisfy:

(i) $(\underset{2}{\operatorname{dim} C+1}) \geqslant \operatorname{dim} \mathcal{C}_{L}(\mathcal{X}, \mathcal{P}, 2 E)$;

(ii) $2 g+1 \leqslant \operatorname{deg}(E) \leqslant \frac{n-2}{2}$;

The left-hand inequality of (iii) is in general satisfied. On the other hand, as explained above, the right-hand inequality of (iii) can be relaxed by using a shortening trick. Constraint (ii) is more central since a subcode which does not satisfies it will probably behave like a random code and it can be checked that a random code is in general 2-closed. Thus, computing the 2-closure of such a subcode will not provide any significant result. On the other hand, for an AG code of dimension $k$, subcodes which do not satisfy (ii) have dimension smaller than $\sqrt{2 k}$ and choosing such very small subcodes and decode them as subcodes of $\mathcal{C}_{L}(\mathcal{X}, \mathcal{P}, E)$ would represent a big loss of efficiency. In addition, if these codes have a too low dimension they can be subject to generic attacks like information set decoding.

\subsection{Subfield subcodes still resist}

Assume $\mathbb{F}_{q}$ to be non prime and let $\mathbb{F}$ be a proper subfield of $\mathbb{F}_{q}$ and $C:=\mathcal{C}_{L}(\mathcal{X}, \mathcal{P}, E) \cap \mathbb{F}^{n}$. The point is that $C^{(2)} \subseteq \mathcal{C}_{L}(\mathcal{X}, \mathcal{P}, E)^{(2)} \cap \mathbb{F}^{n}$ and the 2-closure of $C$ in general differs from $\mathcal{C}_{L}(\mathcal{X}, \mathcal{P}, E)$. For this reason, subfield subcodes resist to this kind of attacks. Notice that even in genus zero: subfield subcodes of GRS codes still resist to filtration attacks unless for the cases presented in [5,10]. Moreover, similarly to the case of classical Goppa codes, some of these codes are known to have a good designed distance, see for instance [18, 43, 47] or [4] for another construction based on the Cartier operator. Therefore, these codes provide a good candidate for a secure generalisation of the original McEliece scheme based on classical Goppa codes. 


\section{Conclusion}

We gave polynomial time algorithms which permit one to reconstruct either an error correcting pair or an error correcting array of a given code. After the works of Faure and Minder [13] who broke McEliece scheme based on codes from hyperelliptic curves of low genus, the present article gives a general attack proving that McEliece scheme based on AG codes from any curve of any genus is totally insecure. Moreover, we show that the countermeasure consisting in replacing an AG code by a random low codimensional subcode is insecure too since the parent code can be recovered by a computation of a 2 -closure.

On the other hand, similarly to the genus 0 case, subfield subcodes of AG codes are completely out of the reach of our attack and remain an interesting candidate for a generalisation of the original McEliece scheme based on classical Goppa codes.

\section{References}

[1] T. Berger and P. Loidreau. How to mask the structure of codes for a cryptographic use. Des. Codes Cryptogr., 35:63-79, 2005.

[2] D. Bernstein. Introduction to post-quantum cryptography. In D. Bernstein, J. Buchmann, and E. Dahmen, editors, Post-quantum cryptography, pages 1-14. Springer-Verlag, Berlin, 2009 .

[3] W. Bosma, J. Cannon, and C. Playoust. The Magma algebra system. I. The user language. J. Symbolic Comput., 24(3-4):235-265, 1997. Computational algebra and number theory (London, 1993).

[4] A. Couvreur. Codes and the cartier operator. Proc. Amer. Math. Soc., 142(6):1983-1996, 2014.

[5] A. Couvreur, O. Ayoub, and T. Jean-Pierre. Polynomial time attack on wild McEliece over quadratic extensions. IEEE Trans. Inform. Theory, 63(1):404-427, Jan 2017.

[6] A. Couvreur, P. Gaborit, V. Gauthier-Umaña, A. Otmani, and J.-P. Tillich. Distinguisherbased attacks on public-key cryptosystems using Reed-Solomon codes. Des. Codes Cryptogr., 73(2):641-666, 2014.

[7] A. Couvreur, I. Márquez-Corbella, and R. Pellikaan. A polynomial time attack against algebraic geometry code based public key cryptosystems. In Proc. IEEE Int. Symposium Inf. Theory - ISIT, pages 1446-1450, 2014.

[8] A. Couvreur, I. Márquez-Corbella, and R. Pellikaan. Cryptanalysis of public-key cryptosystems that use subcodes of algebraic geometry codes. In R. Pinto, P. Malonek, and P. Vettori, editors, 4-th Int. Castle Meeting on Coding Theory and Appl., volume 3 of CIM Series in Mathematical Sciences, pages 133-140. Springer-Verlag Berlin Heidelberg, 2015.

[9] A. Couvreur, A. Otmani, J. Tillich, and V. Gauthier-Umaña. A polynomial-time attack on the BBCRS scheme. In J. Katz, editor, Public-Key Cryptography - PKC 2015, volume 9020 of Lecture Notes in Comput. Sci., pages 175-193. Springer, 2015.

[10] A. Couvreur, A. Otmani, and J.-P. Tillich. Polynomial time attack on wild McEliece over quadratic extensions. In Advances in cryptology-EUROCRYPT 2014, volume 8441 of Lecture Notes in Comput. Sci., pages 17-39. Springer, Heidelberg, 2014. 
[11] I. Duursma. Decoding codes from curves and cyclic codes. PhD thesis, Eindhoven Univ. Technology, 1993.

[12] I. Duursma and R. Kötter. Majority coset decoding. IEEE Trans. Inform. Theory, 39:10671071, 1993.

[13] C. Faure and L. Minder. Cryptanalysis of the McEliece cryptosystem over hyperelliptic codes. In Proc. Int Workshop on Algebraic and Combinatorial Coding Theory, pages 99$107,2008$.

[14] G. Feng and T. Rao. Decoding algebraic-geometric codes up to the designed minimum distance. IEEE Trans. Inform. Theory, 39(1):37-45, 1993.

[15] G. Feng and K. Tzeng. A new procedure for decoding cyclic and BCH codes up to actual minimum distance. IEEE Trans. Inform. Theory, 40(5):1364-1374, 1994.

[16] O. Geil, R. Matsumoto, and D. Ruano. Feng-Rao decoding of primary codes. Finite Fields Appl., 23:35-52, 2013.

[17] H. Janwa and O. Moreno. McEliece public cryptosystem using algebraic-geometric codes. Des. Codes Cryptogr., 8:293-307, 1996.

[18] G. L. Katsman and M. A. Tsfasman. A remark on algebraic geometric codes. In Representation theory, group rings, and coding theory, volume 93 of Contemp. Math., pages 197-199. Amer. Math. Soc., Providence, RI, 1989.

[19] K. Khuri-Makdisi. Linear algebra algorithms for divisors on an algebraic curve. Math. Comp., 73(245):333-357 (electronic), 2004.

[20] K. Khuri-Makdisi. Asymptotically fast group operations on Jacobians of general curves. Math. Comp., 76(260):2213-2239 (electronic), 2007.

[21] C. Kirfel and R. Pellikaan. The minimum distance of codes in an array coming from telescopic semigroups. IEEE Trans. Inform. Theory, 41(6, part 1):1720-1732, 1995. Special issue on algebraic geometry codes.

[22] R. Kötter. A unified description of an error locating procedure for linear codes. In Proc. Int Workshop on Algebraic and Combinatorial Coding Theory, pages 113-117, Voneshta Voda, 1992.

[23] N. Lausten. Design og afkodning af algebraiske geometrikoder. Master's thesis, Technical Univ. of Denmark, Kopenhagen, 1993.

[24] F. J. MacWilliams and N. J. A. Sloane. The Theory of Error-Correcting Codes. NorthHolland, Amsterdam, fifth edition, 1986.

[25] I. Márquez-Corbella, E. Martínez-Moro, and R. Pellikaan. On the unique representation of very strong algebraic geometry codes. Des. Codes Cryptogr., pages 1-16, 2012.

[26] I. Márquez-Corbella, E. Martínez-Moro, and R. Pellikaan. The non-gap sequence of a subcode of a generalized Reed-Solomon code. Des. Codes Cryptogr., 66(1-3):317-333, 2013.

[27] I. Márquez-Corbella, E. Martínez-Moro, R. Pellikaan, and D. Ruano. Computational aspects of retrieving a representation of an algebraic geometry code. J. Symbolic Comput., 64:67-87, 2014. 
[28] R. J. McEliece. A public-key cryptosystem based on algebraic coding theory. DSN Progress Report, 42-44:114-116, 1978.

[29] L. Minder and A. Shokrollahi. Cryptanalysis of the Sidelnikov cryptosystem. In EUROCRYPT 200\%, volume 4515 of Lecture Notes in Comput. Sci., pages 347-360. SpringerVerlag Berlin Heidelberg, 2007.

[30] R. Misoczki, J.-P. Tillich, N. Sendrier, and P. Barreto. MDPC-McEliece: New McEliece variants from moderate density parity-check codes. In Proc. IEEE Int. Symposium Inf. Theory - ISIT, pages 2069-2073, July 2013.

[31] D. Mumford. Varieties defined by quadratic equations. In Questions on algebraic varieties, C.I.M.E., III Ciclo, Varenna, 1969, pages 29-100. Edizioni Cremonese, Rome, 1970.

[32] H. Niederreiter. Knapsack-type cryptosystems and algebraic coding theory. Problems of Control and Information Theory, 15(2):159-166, 1986.

[33] R. Pellikaan. On decoding linear codes by error correcting pairs. Preprint Technical University Eindhoven, 1988.

[34] R. Pellikaan. On decoding by error location and dependent sets of error positions. Discrete Math., 106-107:369-381, 1992.

[35] R. Pellikaan. On the efficient decoding of algebraic-geometric codes. In Eurocode '92 (Udine, 1992), volume 339 of CISM Courses and Lectures, pages 231-253. Springer, Vienna, 1993.

[36] R. Pellikaan. On the existence of error-correcting pairs. Statistical Planning and Inference, 51:229-242, 1996.

[37] C. Peters. Information-set decoding for linear codes over $\mathbb{F}_{q}$. In N. Sendrier, editor, Post-Quantum Cryptography, volume 6061 of Lecture Notes in Comput. Sci., pages 81-94. Springer, 2010.

[38] H. Randriambololona. On products and powers of linear codes under componentwise multiplication. In Algorithmic arithmetic, geometry, and coding theory, volume 637 of Contemp. Math., pages 3-78. Amer. Math. Soc., Providence, RI, 2015.

[39] N. Sendrier. On the structure of a randomly permuted concatenated code. In P. Charpin, editor, EUROCODE '94, pages 169-173, 1994.

[40] P. W. Shor. Algorithms for quantum computation: discrete logarithms and factoring. In Proceedings of the 35th Annual Symposium on Foundations of Computer Science, SFCS '94, pages 124-134. IEEE Computer Society, Washington, DC, USA, 1994.

[41] V. M. Sidelnikov. A public-key crypto system based on binary reed-muller codes. Discrete Math. Appl., 4(3):191-208, 1994.

[42] V. M. Sidelnikov and S. O. Shestakov. On the insecurity of cryptosystems based on generalized Reed-Solomon codes. Discrete Math. Appl., 2:439-444, 1992.

[43] H. Stichtenoth. On the dimension of subfield subcodes. IEEE Trans. Inform. Theory, 36(1):90-93, 1990.

[44] H. Stichtenoth. Algebraic function fields and codes, volume 254 of Graduate Texts in Mathematics. Springer-Verlag, Berlin, second edition, 2009. 
[45] S. Vlădut, D. Nogin, and M. Tsfasman. Algebraic Geometric Codes: Basic Notions, volume 139 of Mathematical Surveys and Monographs. American Mathematical Society, 2007.

[46] C. Wieschebrink. Cryptanalysis of the Niederreiter public key scheme based on GRS subcodes. In Post-Quantum Cryptography, volume 6061 of Lecture Notes in Comput. Sci., pages 61-72. Springer-Verlag Berlin Heidelberg, 2010.

[47] M. Wirtz. On the parameters of Goppa codes. IEEE Trans. Inform. Theory, 34(5, part 2):1341-1343, 1988. Coding techniques and coding theory.

\section{A From degenerate to non-degenerate}

In Section $\S 6.3$, we explain how to compute a subcode $\mathcal{C}_{L}(\mathcal{X}, \mathcal{P}, E-F)$ of $\mathcal{C}_{L}(\mathcal{X}, \mathcal{P}, E)$, however this code is degenerated since $F=(t+g) P$ and hence the position corresponding to $P$ is zero for any codeword of $\mathcal{C}_{L}(\mathcal{X}, \mathcal{P}, E-F)$. This is the reason why we cannot directly perform decoding on $\mathcal{C}_{L}(\mathcal{X}, \mathcal{P}, E)^{\perp}$ and should first decode its puncturing at $P$.

In what follows, we explain how to compute another code $\mathcal{C}_{L}\left(\mathcal{X}, \mathcal{P}, E-F^{\prime}\right)$, where $F^{\prime}$ is linearly equivalent to $F$, i.e. $F^{\prime}=F+(h)$ for some rational and has disjoint support with $D_{\mathcal{P}}$. It should be pointed out that we do not need to compute $h$ but just prove its existence. In addition to the previous calculations, the computation of a generator matrix of $\mathcal{C}_{L}\left(\mathcal{X}, \mathcal{P}, E-F^{\prime}\right)$ requires the knowledge of the code $V_{-(t+g+1)}$ which can be obtained thanks to Proposition 20(ii).

Proposition 27. Let $\mathbf{G}$ be a generator matrix of $V_{-(t+g)}$ of the form

$$
\mathbf{G}=\left(\begin{array}{c|c}
0 & \mathbf{c}_{1} \\
\hline(0) & \mathbf{G}_{1}
\end{array}\right)
$$

where $\mathbf{c}_{1} \in \mathbb{F}_{q}^{n-1}$ and $\left(0 \mid \mathbf{c}_{1}\right) \in V_{-(t+g)} \backslash V_{-(t+g+1)}$ and $\left((0) \mid \mathbf{G}_{1}\right)$ is a generator matrix of $V_{-(t+g+1)}$. Then, there exists a rational function $h$ on $\mathcal{X}$ such that the matrix

$$
\mathbf{G}^{\prime}:=\left(\begin{array}{c|c}
1 & \mathbf{c}_{1} \\
\hline(0) & \mathbf{G}_{1}
\end{array}\right)
$$

is a generator matrix for $\mathcal{C}_{L}(\mathcal{X}, \mathcal{P}, E-(t+g) P-(h))$.

Proof. For simplicity suppose that $P=P_{1}$, i.e. $P$ corresponds to the first column of the generator matrix. Let $f \in L(E-(t+g) P) \backslash L(E-(t+g+1) P)$ be the function such that $\left(0 \mid \mathbf{c}_{1}\right)=\left(f\left(P_{1}\right), \ldots, f\left(P_{n}\right)\right)$. By definition, $v_{P}(f)=t+g$. From the weak approximation Theorem [44, Theorem 1.3.1], there exists a rational function $h \in \mathbb{F}_{q}(\mathcal{X})$ such that

(i) $\forall i \geqslant 2, h\left(P_{i}\right)=1$;

(ii) $v_{P_{1}}(h)=-t-g$ and $h f\left(P_{1}\right)=1$.

Such a function $h$ yields the result. 